\title{
Molecules in bipolar proto-planetary nebulae ${ }^{\star}$
}

\author{
P. M. Woods ${ }^{1,2}$, L.-Å. Nyman ${ }^{2,3}$, F. L. Schöier ${ }^{4}$, A. A. Zijlstra ${ }^{1}$, T. J. Millar ${ }^{1}$, and H. Olofsson ${ }^{4}$ \\ 1 Department of Physics, UMIST, PO Box 88, Manchester M60 1QD, UK \\ e-mail: Tom.Millar@umist.ac.uk \\ 2 European Southern Observatory, Alonso de Cordova 3107, Casilla 19001, Santiago 19, Chile \\ 3 Onsala Space Observatory, 43992 Onsala, Sweden \\ ${ }^{4}$ Stockholm Observatory, AlbaNova, 10691 Stockholm, Sweden
}

Received 9 September 2003 / Accepted 12 July 2004

\begin{abstract}
Two bipolar proto-planetary nebulae, IRAS 16594-4656 and IRAS 17150-3224, have been detected in various molecular lines, namely $\mathrm{CO},{ }^{13} \mathrm{CO}, \mathrm{HCN}$ and $\mathrm{CN}$, and remain undetected in several other species. $\mathrm{CO}(J=2-1)$ and $\mathrm{CO}(J=3-2)$ line profiles are compared to new spectra of similar PPN candidates, previously undetected in $\mathrm{CO}(J=2-1)$ : $\mathrm{CPD}-53^{\circ} 5736$, IRAS 17106-3046, IRAS 17245-3951 and IRAS 17441-2411. CO $(J=2-1)$ maps of IRAS 16594-4656 and IRAS 17150-3224 show that both PPNe have little separation between blue, centre and red-shifted emission, and also that the $\operatorname{CO}(J=2-1)$ emission is of a similar size to the telescope beam. Fractional abundances of all detected molecules (except $\mathrm{CO}$ ) are calculated using the results of CO line modelling and a simple photodissociation model. For those species not detected, upper limits are derived. Comparisons between these fractional abundances and those of other PPNe show that IRAS 16594-4656 and IRAS 17150-3224 are quite under-abundant when compared to molecule-rich sources like CRL618, CRL2688 and OH231.8+4.2. As a reason for this deficit, the difference in circumstellar envelope/torus density between the molecule-rich sources and the molecule-poor sources is proposed, and supported by a chemical model which follows the transition of a circumstellar envelope through the AGB phase and into the PPN phase of evolution. The model includes the effects of UV radiation, cosmic rays and also X-rays. Finally, the post-AGB ages of these two objects (200-400 yr) are estimated using CN/HCN and HCN/CO ratios and both ages are found to be in agreement with previous figures cited in the literature, IRAS 17150-3224 being the younger of the two PPNe.
\end{abstract}

Key words. astrochemistry - stars: AGB and post-AGB - stars: carbon - stars: circumstellar matter stars: individual: IRAS 16594-4656 - stars: individual: IRAS 17150-3224

\section{Introduction}

The proto-planetary stage of evolution is one of the shortest in a star's lifetime and this implies a scarcity of observable examples. Following the method of Olivier et al. (2001), the number surface density of proto-planetary nebulae can be estimated to be $\left(0.41_{-0.34}^{+1.91}\right) \mathrm{kpc}^{-2}$. In comparsion, the density of AGB stars is $15 \mathrm{kpc}^{-2}$ and of Main Sequence stars is $\sim 2 \times 10^{6} \mathrm{kpc}^{-2}$ (Olivier et al. 2001). Hence knowledge about this phase is limited. Most of the current understanding of PPNe is derived from a handful of objects; mainly the carbon-rich sources CRL618 and CRL2688 and the oxygen-rich object $\mathrm{OH} 231.8+4.2$. All three show strong molecular lines, have axisymmetric structures and molecular tori or disks. However, several hundred PPNe candidates have been identified (see Kwok 1993, and references therein), some 34 are reasonably well identified as PPNe (Bujarrabal et al. 2001), and yet only a handful show such molecular "richness". The reason for this difference is far from clear.

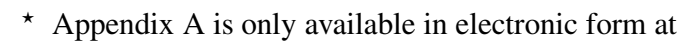
http://www.edpsciences.org
Many optical and infrared HST images have helped in the study of PPNe (e.g., see Su et al. 2001; Hrivnak et al. 2001, 1999; Kwok et al. 1998, for details on the two PPNe described here), although the depth to which many of the objects discovered have been studied is minimal. Both molecule-rich PPNe and molecule-poor PPNe appear similar in images - the three PPNe mentioned above and both IRAS 16594-4656 and IRAS 17150-3224 have some degree of bipolarity, and a narrow waist. The beginnings of this bipolarity are found in the late AGB phase, where the first signs of asphericity are seen (e.g., Kastner \& Weintraub 1994). The shaping of the nebula continues under the influence of a fast superwind, according to the Generalized Interacting Stellar Wind (GISW) model (Balick 1987). Other effects then play a part in the developing morphology (see the review by Balick \& Frank 2002), and one which may be important is the inertial confinement of the outflowing wind by a circumstellar torus or disk (Calvet \& Peimbert 1983). The degree of collimation produced by this disk would depend on the mass of the disk, as well as the momentum involved in the high-speed outflow, and other, geometrical effects. This change in morphology, from something approximately 
spherical to something bipolar, or elliptical, occurs very rapidly at the end of the AGB phase of evolution (e.g., Kwok et al. 1996; Schmidt et al. 2002). The actual period of transition is hard to quantify, and there are inherent difficulties in estimates by dynamical means (Zijlstra et al. 2001).

In this paper, two objects which have been imaged by the HST and show strong CO emission are studied. Both objects, IRAS 16594-4656 and IRAS 17150-3224 are only detected in a handful of species, including $\mathrm{HCN}$ and $\mathrm{CN}$. These are compared to other PPNe newly observed in $\mathrm{HCN}$ and $\mathrm{CO}$ - CPD-53 5736, IRAS 17106-3046, IRAS 17245-3951 and IRAS 17441-2411 (Sect. 4). Furthermore, the molecular properties of these two PPNe are studied by calculating fractional abundances from SEST data, and comparing with similar objects (Sects. 6.2.1 and 7). The comparative under-abundance of molecules in IRAS 16594-4656 and IRAS 17150-3224 is discussed in Sect. 7, and arising hypotheses are confirmed by means of a chemical model (Sect. 6.3). Finally, individual spectra are presented in Appendix A, which is only available in electronic form.

\section{Observations}

The observations were carried out between 1998 and 2002 with the Swedish-ESO Submillimetre Telescope (SEST), situated on La Silla, Chile. SIS receivers were used at 0.8, 1.3, 2 and $3 \mathrm{~mm}$. During this period the SEST operated three acoustooptical spectrometers: one high resolution spectrometer, with a bandwidth of $86 \mathrm{MHz}$ and a channel separation of $43 \mathrm{kHz}$, and two wideband spectrometers with bandwidths of about $1 \mathrm{GHz}$ and a channel separation of $0.7 \mathrm{MHz}$. Typical system temperatures above the atmosphere ranged between 150 and $800 \mathrm{~K}$, depending on frequency and elevation.

Most observations were carried out with the dual beam switching method which gives very flat baselines by placing the source alternately in two beams. Beam separation was 11.5 in azimuth. CO maps of IRAS 17150-3224 were taken using the position switching method, to minimise interference from interstellar $\mathrm{CO}$ emission lines. The OFF position was chosen to be $\left(-10^{\prime},-11^{\prime}\right)$ from the $\mathrm{ON}$ position. Map spacing in all cases was $11^{\prime \prime}$, which is approximately half a beamwidth at $230 \mathrm{GHz}$. Calibration was performed with the standard chopper-wheel technique, and has an uncertainty of approximately $20 \%$ (see Schöier \& Olofsson 2001, for details). J2000 positions of both sources are given in Table 1.

Intensity scales of spectra in this paper are given in main-beam brightness temperature, which is the corrected antenna temperature divided by the main-beam efficiency $\left(\eta_{\mathrm{mb}}\right)$. Beam efficiencies and $F W H M$ beam widths are summarised in Table 2. Individual spectra are shown in Appendix A, and line frequencies and integrated intensities are shown in Table 3.

Integrated intensities of lines which were not clearly detected are calculated using the following expression,

$I_{v} \leq 3 \sigma\left(\sqrt{2} \sqrt{2 \frac{v_{\text {exp }}}{\Delta v_{\text {res }}}}\right) \Delta v_{\text {res }}=3 \sigma(2 n)^{1 / 2} \Delta v_{\text {res }}$,

where $\sigma$ is the rms noise in the spectrum, $v_{\exp }$ the expansion velocity, $\Delta v_{\text {res }}$ the velocity resolution of the spectrum and $n$
Table 1. Positions and LSR velocities for the PPNe under study.

\begin{tabular}{lcccc}
\hline \hline IRAS No. & Other name & \multicolumn{2}{c}{ J2000 Co-ords. } & $v_{\text {LSR }}$ \\
& & {$[\mathrm{h}: \mathrm{m}: \mathrm{s}]$} & {$\left[:^{\prime}:^{\prime \prime}\right]$} & {$\left[\mathrm{km} \mathrm{s}^{-1}\right]$} \\
\hline $14488-5405$ & CPD-53 5736 & $14: 52: 28.7$ & $-54: 17: 43$ & -10 \\
$16594-4656$ & - & $17: 03: 09.7$ & $-47: 00: 28$ & -25 \\
$17106-3046$ & - & $17: 13: 51.7$ & $-30: 49: 40$ & 0 \\
$17150-3224$ & AFGL6815S & $17: 18: 20.0$ & $-32: 27: 20$ & +15 \\
$17245-3951$ & OH348.8-2.8 & $17: 28: 04.8$ & $-39: 53: 44$ & 0 \\
$17441-2411$ & AFGL5385 & $17: 47: 10.3$ & $-24: 12: 54$ & +110 \\
\hline
\end{tabular}

Table 2. SEST FWHM beam widths and efficiencies at selected frequencies.

\begin{tabular}{ccc}
\hline \hline $\begin{array}{c}\text { Frequency } \\
\text { [GHz] }\end{array}$ & $\begin{array}{c}F W H M \\
{\left[{ }^{\prime \prime}\right]}\end{array}$ & $\eta_{\mathrm{mb}}$ \\
\hline 86 & 57 & 0.75 \\
100 & 51 & 0.73 \\
115 & 45 & 0.70 \\
147 & 34 & 0.66 \\
230 & 23 & 0.50 \\
265 & 21 & 0.42 \\
345 & 15 & 0.25 \\
\hline
\end{tabular}

the number of channels covering the line width. This gives a somewhat optimistic view of the integrated intensity of the line, and hence any fractional abundance calculation using this value will be strictly an upper limit. Values of $I_{v}$ calculated in this manner are given in Table 3 and indicated by a "less than" sign.

\section{Sources}

The two PPNe presented in this paper are objects for which there are currently few millimetre-wavelength spectra and little or no molecular information. Both show bipolar morphology in HST images and also bright $\mathrm{CO}$ emission, with interesting features. These two sources were selected on the basis of previously published CO spectra (Loup et al. 1990; Hu et al. 1993).

\subsection{IRAS 16594-4656}

IRAS 16594-4656 is a proto-planetary nebula which lies almost in the plane of the Galaxy $(b=-3.3$, van de Steene \& van Hoof 2003) and has a mixed chemistry. In its infrared spectrum there are strong and rarely observed features at 12.6 and $13.4 \mu \mathrm{m}$, thought to be due to polycyclic aromatic hydrocarbons (PAHs) with a high degree of hydrogenation, and further PAH features in the range 3-13 $\mu \mathrm{m}$ (García-Lario et al. 1999). An emission feature between 19 and $23 \mu \mathrm{m}$, associated with C-rich PPNe by Kwok \& Hrivnak (1989), also suggests that this source is carbon rich. However, there are also indications of crystalline silicates (pyroxenes), with weak features around $34 \mu \mathrm{m}$ (García-Lario et al. 1999), and a $10 \mu \mathrm{m}$ silicate feature (Olnon et al. 1986). This suggests that until recently this object was oxygen-rich, becoming carbon-rich shortly before the transition to the post-AGB phase (García-Lario et al. 1999), which occurred some 370 years ago (van der Veen et al. 1989). 
Table 3. Observed lines.

\begin{tabular}{|c|c|c|c|c|c|c|}
\hline \multirow[b]{2}{*}{ Molecule } & \multirow[b]{2}{*}{ Transition } & \multirow[b]{2}{*}{$\begin{array}{l}\text { Frequency } \\
\mathrm{GHz}\end{array}$} & \multicolumn{2}{|c|}{ IRAS 16594-4656 } & \multicolumn{2}{|c|}{ IRAS 17150-3224 } \\
\hline & & & $\begin{array}{c}T_{\mathrm{mb}} \\
\mathrm{K}\end{array}$ & $\begin{array}{c}\int T_{\mathrm{mb}} \mathrm{d} v \\
\mathrm{~K} \mathrm{~km} \mathrm{~s}^{-1}\end{array}$ & $\begin{array}{c}T_{\mathrm{mb}} \\
\mathrm{K}\end{array}$ & $\begin{array}{c}\int T_{\mathrm{mb}} \mathrm{d} v \\
\mathrm{~K} \mathrm{~km} \mathrm{~s}^{-1}\end{array}$ \\
\hline $\mathrm{C}_{3} \mathrm{~S}$ & $J=15-14$ & 86.708 & $<0.01$ & $<0.03$ & - & - \\
\hline $\mathrm{SiO}$ & $J=2-1$ & 86.847 & $<0.01$ & $<0.03$ & - & - \\
\hline $\mathrm{HN}^{13} \mathrm{C}$ & $J=1-0$ & 87.091 & $<0.01$ & $<0.03$ & - & - \\
\hline $\mathrm{C}_{4} \mathrm{H}$ & ${ }^{2} \Pi_{3 / 2}, 19 / 2-17 / 2$ & 87.372 & $<0.01$ & $<0.03$ & - & - \\
\hline $\mathrm{C}_{2} \mathrm{H}$ & $N=1-0$ & 87.329 & $<0.01$ & $<0.03$ & - & - \\
\hline $\mathrm{HCN}$ & $J=1-0$ & 88.632 & 0.02 & 0.47 & 0.02 & 0.54 \\
\hline $\mathrm{C}_{3} \mathrm{~N}$ & $N=9-8, J=19 / 2-17 / 2$ & 89.046 & $<0.01$ & $<0.05$ & - & - \\
\hline $\mathrm{C}_{3} \mathrm{~N}$ & $N=9-8, J=17 / 2-15 / 2$ & 89.064 & $<0.01$ & $<0.05$ & - & - \\
\hline $\mathrm{HCO}^{+}$ & $J=1-0$ & 89.189 & $<0.01$ & $<0.05$ & - & - \\
\hline $\mathrm{HC}^{13} \mathrm{CCN}$ & $J=10-9$ & 90.593 & $<0.01$ & $<0.04$ & - & - \\
\hline $\mathrm{HCC}^{13} \mathrm{CN}$ & $J=10-9$ & 90.602 & $<0.01$ & $<0.04$ & - & - \\
\hline HNC & $J=1-0$ & 90.664 & $<0.01$ & $<0.04$ & - & - \\
\hline $\mathrm{SiS}$ & $J=5-4$ & 90.772 & $<0.01$ & $<0.04$ & - & - \\
\hline $\mathrm{HC}_{3} \mathrm{~N}$ & $J=10-9$ & 90.979 & $<0.01$ & $<0.04$ & - & - \\
\hline $\mathrm{CH}_{3} \mathrm{CN}$ & $5(1)-4(1)$ & 91.985 & $<0.01$ & $<0.05$ & - & - \\
\hline $\mathrm{HC}^{13} \mathrm{CCN}$ & $J=12-11$ & 108.711 & $<0.01$ & $<0.06$ & - & - \\
\hline $\mathrm{HCC}^{13} \mathrm{CN}$ & $J=12-11$ & 108.721 & $<0.01$ & $<0.06$ & - & - \\
\hline${ }^{13} \mathrm{CN}$ & $N=1-0$ & 108.780 & $<0.01$ & $<0.06$ & - & - \\
\hline $\mathrm{C}_{3} \mathrm{~N}$ & $N=11-10, J=23 / 2-21 / 2$ & 108.834 & $<0.01$ & $<0.06$ & - & - \\
\hline $\mathrm{C}_{3} \mathrm{~N}$ & $N=11-10, J=21 / 2-19 / 2$ & 108.853 & $<0.01$ & $<0.06$ & - & - \\
\hline $\mathrm{SiS}$ & $J=6-5$ & 108.924 & $<0.01$ & $<0.06$ & - & - \\
\hline $\mathrm{HC}_{3} \mathrm{~N}$ & $J=12-11$ & 109.174 & $<0.01$ & $<0.06$ & - & - \\
\hline${ }^{13} \mathrm{CO}$ & $J=1-0$ & 110.201 & 0.04 & 0.06 & 0.04 & 0.75 \\
\hline $\mathrm{CH}_{3} \mathrm{CN}$ & $6(1)-5(1)$ & 110.381 & $<0.01$ & $<0.11$ & $<0.01$ & $<0.09$ \\
\hline $\mathrm{C}_{4} \mathrm{H}$ & ${ }^{2} \Pi_{3 / 2} 23 / 2-21 / 2$ & 113.266 & $<0.01$ & $<0.09$ & $<0.01$ & $<0.06$ \\
\hline $\mathrm{C}_{2} \mathrm{~S}$ & $8(7)-9(8)$ & 113.410 & $<0.01$ & $<0.09$ & $<0.01$ & $<0.06$ \\
\hline $\mathrm{CN}$ & $N=1-0$ & 113.491 & 0.05 & 3.63 & $<0.01$ & $<0.06$ \\
\hline $\mathrm{C}_{4} \mathrm{H}$ & ${ }^{2} \Pi_{3 / 2} 21 / 2-19 / 2$ & 115.217 & $<0.07$ & $<0.76$ & $<0.04$ & $<0.45$ \\
\hline $\mathrm{CO}$ & $J=1-0$ & 115.271 & 0.61 & 1.78 & 0.25 & 5.05 \\
\hline $\mathrm{SiC}_{2}$ & $5_{0,5}-4_{0,4}$ & 115.382 & $<0.07$ & $<0.76$ & $<0.04$ & $<0.45$ \\
\hline $\mathrm{SiO}$ & $J=3-2$ & 130.269 & $<0.01$ & $<0.06$ & - & - \\
\hline $\mathrm{H}_{2} \mathrm{CO}$ & $2_{1,2}-1_{1,1}$ & 140.840 & $<0.01$ & $<0.07$ & - & - \\
\hline $\mathrm{SiC}_{2}$ & $6_{2,5}-5_{2,4}$ & 140.920 & $<0.01$ & $<0.07$ & - & - \\
\hline $\mathrm{H}^{13} \mathrm{CCCN}$ & $J=16-15$ & 141.062 & $<0.01$ & $<0.07$ & - & - \\
\hline CS & $J=3-2$ & 146.969 & $<0.01$ & $<0.04$ & - & - \\
\hline $\mathrm{CH}_{3} \mathrm{CN}$ & $8(0)-7(0)$ & 147.175 & $<0.01$ & $<0.04$ & - & - \\
\hline${ }^{13} \mathrm{CO}$ & $J=2-1$ & 220.399 & 0.10 & 1.34 & 0.13 & 2.91 \\
\hline $\mathrm{CH}_{3} \mathrm{CN}$ & $12(0)-11(0)$ & 220.747 & $<0.01$ & $<0.16$ & $<0.01$ & $<0.17$ \\
\hline $\mathrm{CN}$ & $N=2-1$ & 226.875 & 0.11 & 9.91 & $<0.01$ & $<0.10$ \\
\hline $\mathrm{CO}$ & $J=2-1$ & 230.538 & 1.75 & 32.60 & 0.65 & 12.84 \\
\hline $\mathrm{CO}^{+}$ & $J=2-1$ & 235.790 & $<0.01$ & $<0.12$ & - & - \\
\hline $\mathrm{SiS}$ & $J=13-12$ & 235.961 & $<0.01$ & $<0.12$ & - & - \\
\hline $\mathrm{CH}_{3} \mathrm{CN}$ & $13(0)-12(0)$ & 239.138 & $<0.01$ & $<0.16$ & - & - \\
\hline $\mathrm{CH}_{3} \mathrm{C}_{2} \mathrm{H}$ & $14(0)-13(0)$ & 239.252 & $<0.01$ & $<0.16$ & - & - \\
\hline $\mathrm{C}_{3} \mathrm{H}_{2}$ & $4_{4,1}-3_{3,0}$ & 265.759 & $<0.02$ & $<0.36$ & - & - \\
\hline $\mathrm{HCN}$ & $J=3-2$ & 265.886 & 0.11 & 2.85 & - & - \\
\hline $\mathrm{H}^{13} \mathrm{CN}$ & $J=4-3$ & 345.340 & $<0.04$ & $<0.80$ & $<0.06$ & $<1.28$ \\
\hline $\mathrm{HC}_{3} \mathrm{~N}$ & $J=38-37$ & 345.610 & $<0.04$ & $<0.80$ & $<0.06$ & $<1.28$ \\
\hline $\mathrm{CO}$ & $J=3-2$ & 345.796 & 2.05 & 41.20 & 0.78 & 18.19 \\
\hline
\end{tabular}


This hypothesis was strengthened by the lack of a detection in OH (te Lintel Hekkert et al. 1991; Silva et al. 1993) and no $\mathrm{SiO}$ maser emission (Nyman et al. 1998). In evolved stars with a mixed chemistry such as this, the silicates are found in a torus and the PAHs in the polar flow (Matsuura et al. 2004).

Several authors describe HST images of this PPN (Hrivnak et al. 1999, 2000; García-Lario et al. 1999), which show the presence of a bright, B7-type (van de Steene et al. 2000) central star, surrounded by a multiple-axis bipolar nebulosity with a complex morphology. The nebula is seen at an intermediate inclination, rather than edge-on. The optical size of the nebula is given as $5^{\prime \prime} \times 11^{\prime \prime}$ (García-Lario et al. 1999).

The only $\mathrm{CO}$ spectrum of this source previously published is a CO $(J=1-0)$ spectrum from Loup et al. (1990), who detected broad $\mathrm{CO}$ emission corresponding to that from a circumstellar shell expanding with a velocity of $16 \mathrm{~km} \mathrm{~s}^{-1}$.

\subsection{IRAS 17150-3224}

IRAS 17150-3224 (AFGL6815S) is a young PPN which left the AGB some 150 ( $\mathrm{Hu}$ et al. 1993) to 210 years ago, and possibly came from a high-mass progenitor (Meixner et al. 2002). Note however, that van der Veen et al. (1989) give a dynamic timescale of $800 \mathrm{yr}$ for this object. Structurally, it is a bipolar nebula, with a nearly edge-on $\left(82^{\circ}\right)$ dusty torus (Kwok et al. 1996), expanding at $11 \mathrm{~km} \mathrm{~s}^{-1}$ (Weintraub et al. 1998) around a G2-type central star. The optical size of the nebula is approximately $12^{\prime \prime} \times 9^{\prime \prime}$ (Hu et al. 1993; Kwok et al. 1996; García-Hernández et al. 2002). A newly-discovered equatorial loop seen in near-infrared, but not optical, images, is discussed by Su et al. (2003). A faint halo (AGB mass-loss remnant) can be seen in images from Kwok et al. (1998).

IRAS 17150-3224 shows molecular hydrogen emission, but not hydrogen recombination, indicating that the degree of ionisation is small (García-Hernández et al. 2002). The emission is likely due to shock-excitation, as is often seen in strongly bipolar nebulae. This object is similar to CRL2688 (Sahai et al. 1998) in that it has $\mathrm{H}_{2}$ emission, and a spectral type later than A (CRL2688 has a spectral type of F2, Cohen \& Kuhi 1977). IRAS 17150-3224 shows OH maser emission (Hu et al. 1994), but not $\mathrm{SiO}$ maser emission (Nyman et al. 1998). It also has been detected in the $3.1 \mu \mathrm{m}$ water line (van der Veen et al. 1989), but not in $\mathrm{H}_{2} \mathrm{O}$ maser emission (Zuckerman \& Lo 1987).

Previous $\mathrm{CO}$ observations are limited to the paper of $\mathrm{Hu}$ et al. (1993), who present both CO $(J=1-0)$ and $(J=2-1)$ spectra.

\section{CO observations}

\subsection{IRAS 16594-4656}

The CO $(J=2-1)$ and $(J=3-2)$ line profiles presented in Fig. 1a are affected by interstellar emission in the OFF position (between -12 and $-5 \mathrm{~km} \mathrm{~s}^{-1}$ approximately). The $\mathrm{CO}$ $(J=3-2)$ profile is taken in dual beamswitch mode whereas the $\mathrm{CO}(J=2-1)$ profile was taken in position switch mode with an OFF position $-30^{\prime \prime}$ away in RA in order to minimise the effect of the interstellar line. Despite this, a high signal-tonoise ratio affords some indication of structure in this source, shown by features in the line profile. A roughly parabolic profile and a source size comparable to the beam shows that the $\mathrm{CO}$ emission is probably optically thick. An outflowing wind of $14 \mathrm{~km} \mathrm{~s}^{-1}$ is indicated by the line profile, and there seems to be an appreciable line wing on the blue side, possibly indicating a second wind of up to $25 \mathrm{~km} \mathrm{~s}^{-1}$. The $\mathrm{CO}(J=2-1)$ map of this source (Fig. 3) shows a reasonably symmetric pattern of emission. The red/blue-shifted emission contour plot (Fig. 2) does not show the red-shifted emission, due to the interstellar interference. The blue-shifted emission, taken in the interval $[-50,-35] \mathrm{km} \mathrm{s}^{-1}$, lies directly on top of the centre emission, taken in the interval $[-35,-12] \mathrm{km} \mathrm{s}^{-1}$, indicating that there is little or no separation in emission regions. There is some emission at $\pm 20^{\prime \prime}$ in RA, and an elliptical Gaussian fit to the integrated intensity map shows that the $F W H M$ ellipse has a major axis of $\sim 25^{\prime \prime}$ and a minor axis of $\sim 22.5^{\prime \prime}$ (position angle of $57^{\circ}$ ), very similar to the size of the SEST beam $\left(23^{\prime \prime}\right)$ at $230 \mathrm{GHz}$. Hence the source is on the limit of being resolved.

\subsection{IRAS $17150-3224$}

The CO $(J=2-1)$ spectrum (Fig. 1b) of IRAS 17150-3224 was taken in position switch mode, with an OFF position of $+45^{\prime \prime}$ in RA. The $\mathrm{CO}(J=3-2)$ spectrum was taken in beamswitch mode. Both CO line profiles of IRAS 17150-3224 shown in Fig. 1b are distinctly triangular in shape, similar to those shown by 89 Her, the Red Rectangle, and M 2-9 (and also IRAS 17441-2411, presented in this paper). Here the triangular shape is affected by an interstellar line in the blue-shifted wing (between 0 and $10 \mathrm{~km} \mathrm{~s}^{-1}$ approximately). The CO lines indicate a wind of some $30 \mathrm{~km} \mathrm{~s}^{-1}$ coming from the source, and yet a parabolic fit to the line indicates a wind of $14.5 \mathrm{~km} \mathrm{~s}^{-1}$. This latter wind is ostensibly the AGB wind of the star, with the higher velocity emission probably coming from an inner swept-up shell, moving at a velocity intermediate to the AGB wind and a post-AGB or super-wind. It must be noted, however, that a parabolic fit to a triangular lineshape will usually underestimate the expansion velocity of the outflowing wind.

The CO ( $J=2-1)$ emission map (Fig. 4) is taken in position switch mode to minimise the effects of the interstellar interference, and although hampered slightly by poor pointing, seems to show a reasonably symmetric distribution which does not appear to be resolved by the telescope beam. The contour map (Fig. 2) does not show any separation in the source. The red-shifted emission was taken from the interval $[30,40] \mathrm{km} \mathrm{s}^{-1}$, the centre emission from $[5,30] \mathrm{km} \mathrm{s}^{-1}$ and the blue-shifted emission from $[-18,-6] \mathrm{km} \mathrm{s}^{-1}$. A twodimensional elliptical Gaussian fit to the centre emission gives an extent of $\sim 20^{\prime \prime} \times 26^{\prime \prime}$ (position angle of $-160^{\circ}$ ) for this source. Again, this is similar to the size of the SEST beam at $230 \mathrm{GHz}$. 
P. M. Woods et al.: Molecules in bipolar PPNe

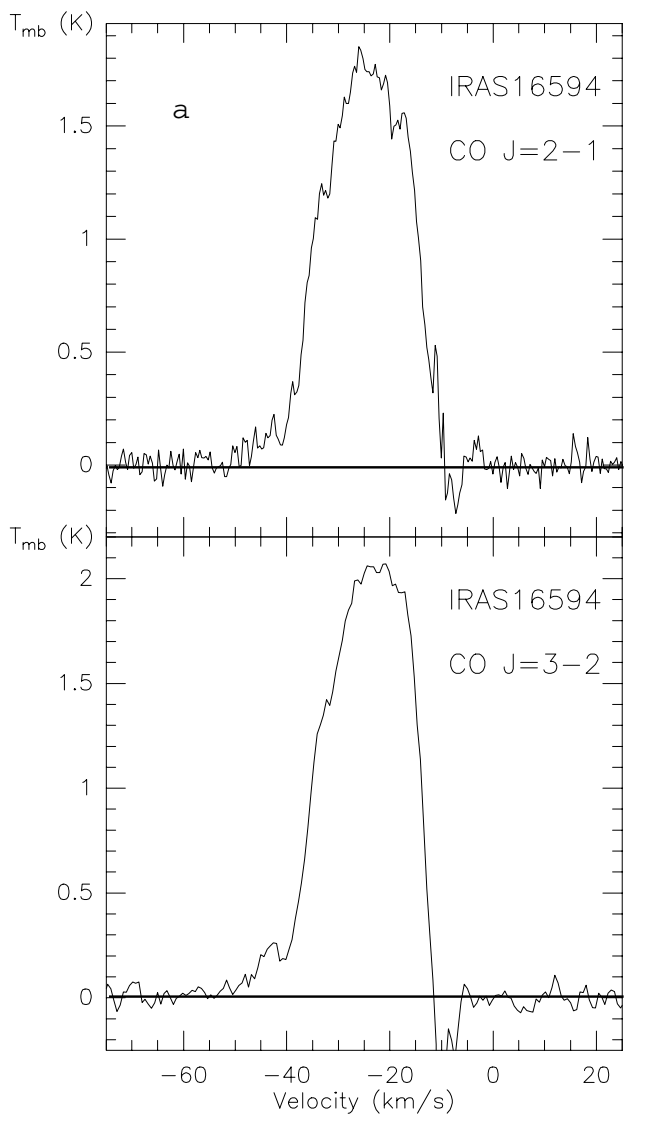

$16594-4656$

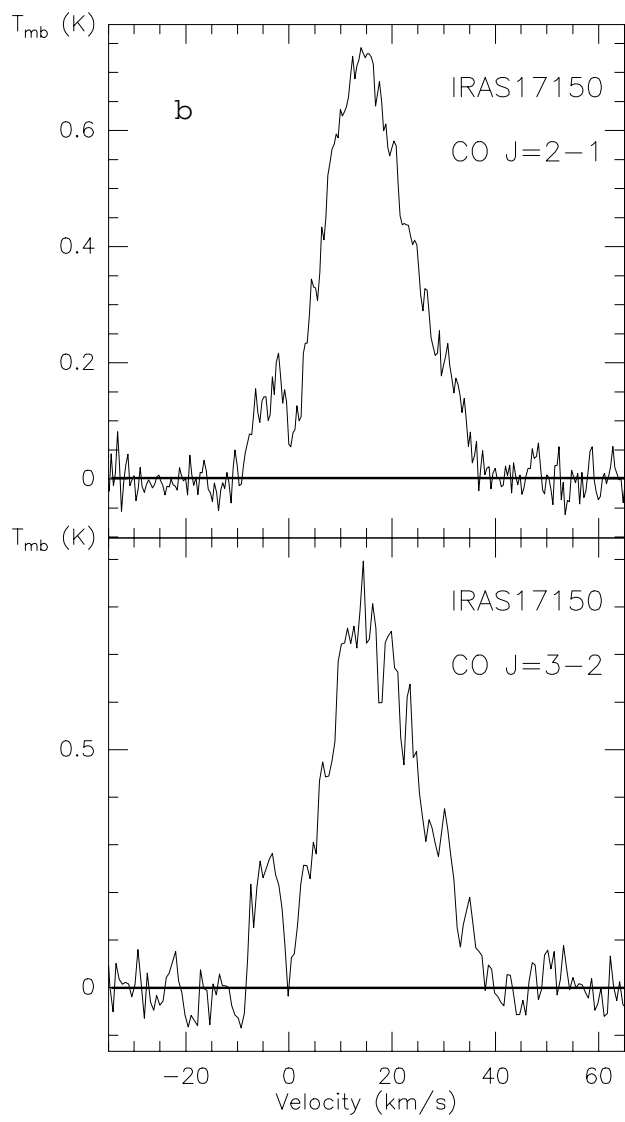

Fig. 1. a) Top. A position-switched high-resolution CO $J=2-1$ spectrum toward IRAS 16594-4656. Bottom. A beam-switched CO $J=$ 3-2 spectrum toward IRAS 165944656. b) Top. A position-switched high-resolution $\mathrm{CO} J=2-1$ spectrum toward IRAS 17150-3224. Bottom. A beam-switched CO $J=$ 3-2 spectrum toward IRAS 171503224.

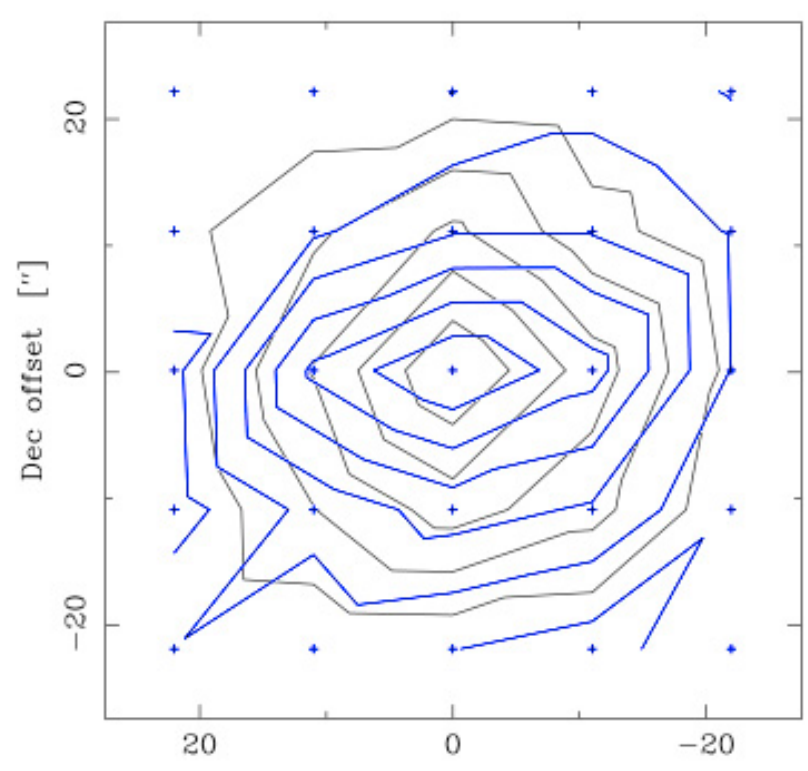

RA offset ["]

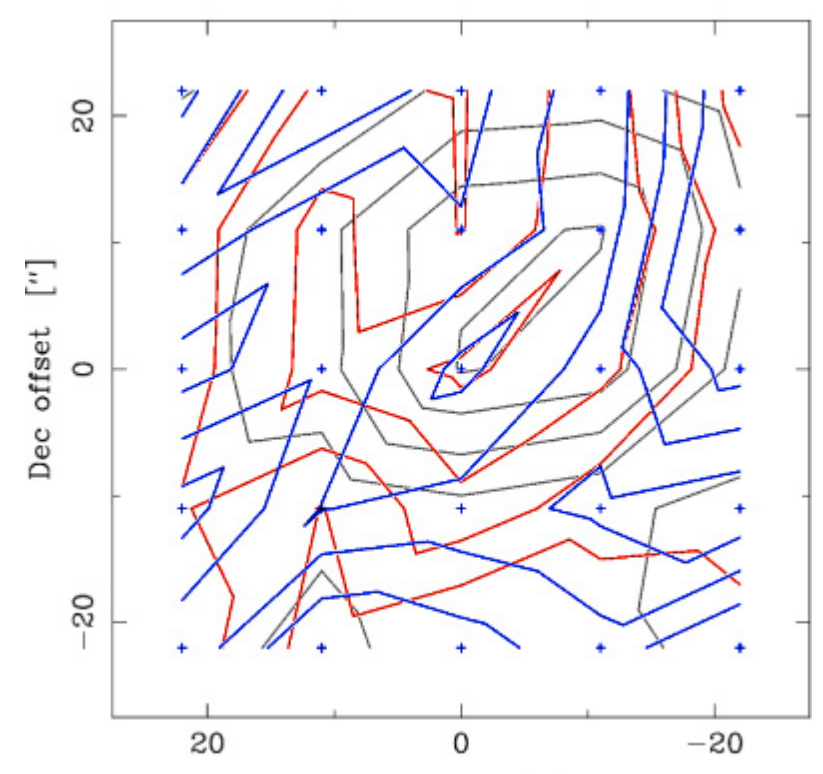

RA offset ["]

Fig. 2. Position-position contour emission maps of IRAS 16594-4656, and IRAS 17150-3224. Blue contours show blue-shifted emission, black contours emission from the centre of the line profile, and red contours red-shifted emission.

\subsection{Other sources observed in CO}

Spectra of select sources which have been hitherto unobserved and/or unpublished in the CO $(J=2-1)$ line are presented in Fig. 5. HCN $(J=1-0)$ spectra have also been taken (not presented), and $\mathrm{HCN} / \mathrm{CO}$ intensity ratios are derived (Table 4).

$C P D-53^{\circ} 5736$. Although not clear from the noisy spectrum, CPD-53 5736 seems to show the presence of a wind expanding at $15 \mathrm{~km} \mathrm{~s}^{-1}$. 


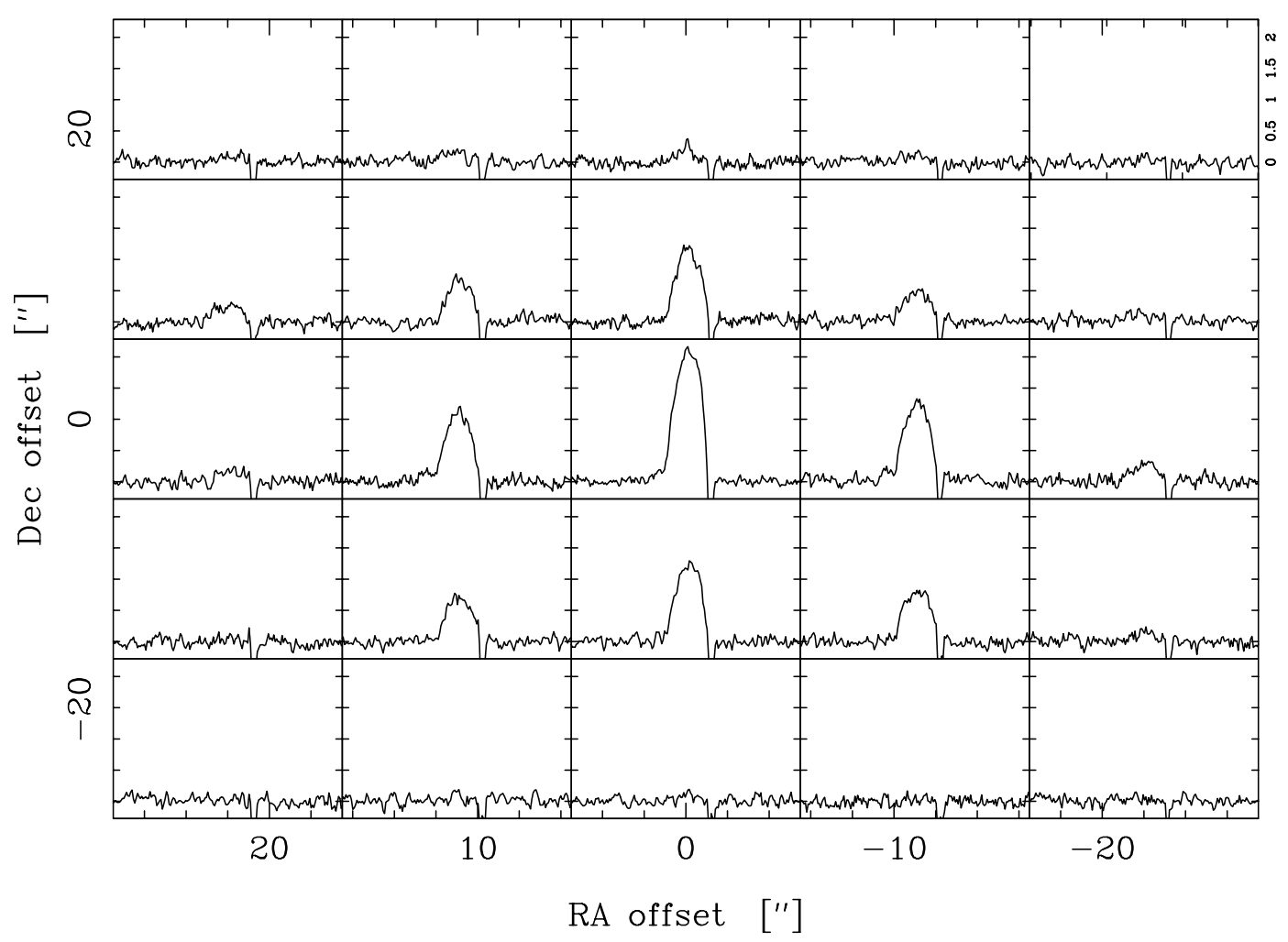

Fig. 3. A map of the CO $J=2-1$ line around IRAS 16954-4656. The beam-spacing is $11^{\prime \prime}$. The spectra are affected by interstellar line contamination in the red-shifted line wing.

IRAS 17106-3046. This object shows a strange profile: some low-level emission, perhaps a wind of $\sim 15 \mathrm{~km} \mathrm{~s}^{-1}$, and a thin, narrow spike. This narrow spike (of width $\sim 2 \mathrm{~km} \mathrm{~s}^{-1}$ ) could correspond in some way to the circumstellar disk observed by Kwok et al. (2000). The narrow feature seems to be offset from the centre of the broad feature, although the signalto-noise ratio is low.

IRAS 17245-3951. Low signal-to-noise means that this profile is hard to qualify. The profile suggests an expansion velocity of $15 \mathrm{~km} \mathrm{~s}^{-1}$, but this value does have a considerable uncertainty.

IRAS 17441-2411. This source has a triangular lineshape, similar to IRAS 17150-3224. It is somewhat asymmetric, and quite narrow in velocity $\left(20 \mathrm{~km} \mathrm{~s}^{-1}\right.$ in width). There are no appreciable line wings.

\subsection{1. $I_{\mathrm{HCN}} / I_{\mathrm{CO}}$ ratios}

The $I_{\mathrm{HCN}} / I_{\mathrm{CO}}$ ratios shown in Table $4(\sim 0.01-0.05)$ look to be reasonably consistent throughout the sample of PPN candidates. To put these figures into context, a sample of oxygen stars selected by Lindqvist et al. (1988) has an average $I_{\mathrm{HCN}} / I_{\mathrm{CO}}$ ratio of 0.124 , whereas the carbon star sample of Olofsson et al. (1990) has an average $I_{\mathrm{HCN}} / I_{\mathrm{CO}}$ ratio of 0.623 . Both these samples were made up of AGB stars; the values for PPNe seem to be smaller. IRAS $07134+1005$ and IRAS 195001709 , both C-rich PPNe, have $I_{\mathrm{HCN}} / I_{\mathrm{CO}}$ ratios of 0.15 and 0.08 , respectively (Bujarrabal et al. 1992). Evolved PNe generally have $I_{\mathrm{HCN}} / I_{\mathrm{CO}}$ ratios of less than 0.1 (cf., Bachiller et al. 1997a).

\section{CO line modelling}

A detailed, non-LTE, radiative transfer code based on the Monte Carlo method was used in order to model the observed molecular line emission. The code assumes the emitting envelope to be spherically symmetric and expanding at a constant velocity. The thermal balance equation, where $\mathrm{CO}$ is assumed to be the main molecular coolant, is solved together with the molecular excitation in order to obtain the kinetic temperature structure. The $\mathrm{CO}$ observations are used as constraints in the modelling to determine also the density and velocity fields through a $\chi^{2}$-analysis. This approach was also adopted by Woods et al. (2003b) when modelling a sample of high mass-loss rate carbon stars. More details on the radiative transfer modelling can be found in Schöier \& Olofsson (2001). The code has been benchmarked to a high accuracy (van Zadelhoff et al. 2002).

In order to derive the mass loss rate which, together with the expansion velocity, sets the density scale in the wind through the continuity equation, a $\mathrm{CO}$ abundance relative to $\mathrm{H}_{2}$ must be adopted. For IRAS $16594-4656$ a value of $1 \times 10^{-3}$, which is typical of a C-rich AGB envelope, is used, whereas for the O-rich object IRAS $17150-3224$ a value of $2 \times 10^{-4}$ is adopted. The derived envelope properties such as the mass loss rate $(\dot{M})$, expansion velocity $\left(v_{\text {exp }}\right)$, extent of the $\mathrm{CO}$ envelope 


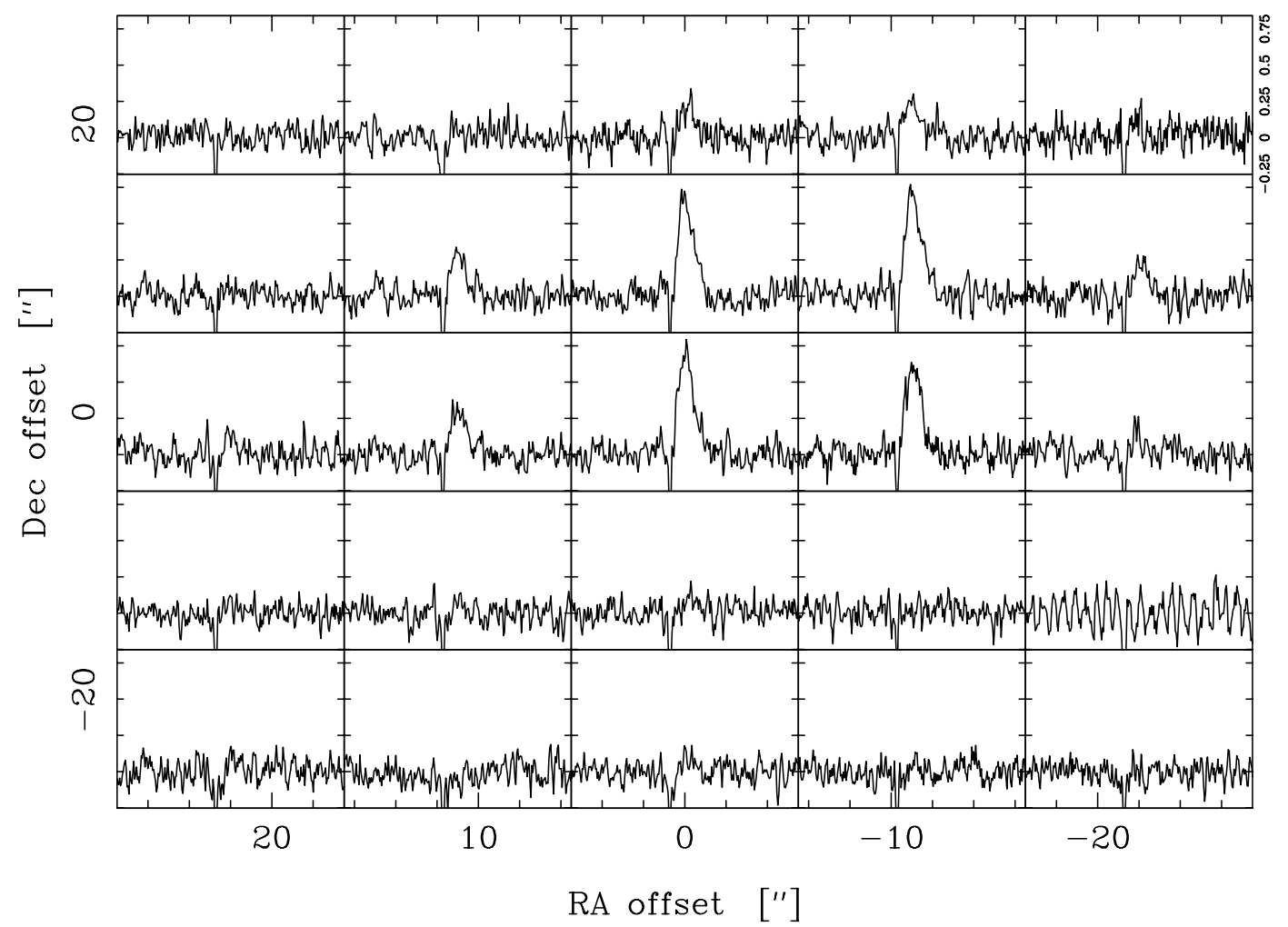

Fig. 4. A map of the CO $J=2-1$ line around IRAS 17150-3224, taken in beamswitch mode. The beam-spacing is $11^{\prime \prime}$. The spectra are affected by interstellar line contamination in the blue-shifted line wing.
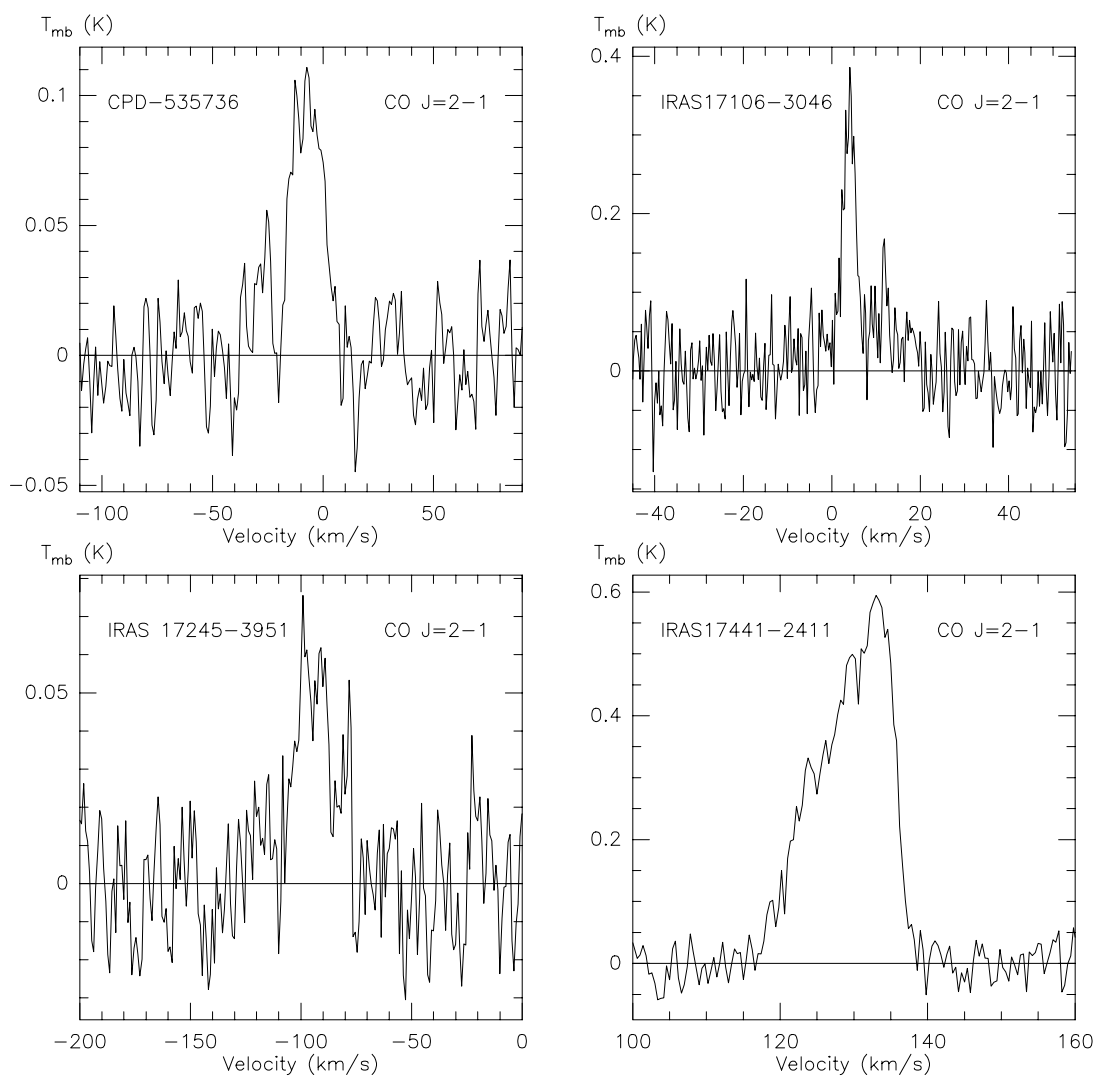

Fig. 5. $\mathrm{CO} J=2-1$ spectra of PPNe candidates, named in the upper left-hand corner of each spectrum. 
Table 4. Observed lines.

\begin{tabular}{|c|c|c|c|c|c|c|c|c|c|c|c|c|}
\hline \multirow{3}{*}{ Molecule } & \multicolumn{2}{|c|}{ CPD-535 5736} & \multicolumn{2}{|c|}{ IRAS 16594-4656 } & \multicolumn{2}{|c|}{ IRAS 17106-3046 } & \multicolumn{2}{|c|}{ IRAS 17150-3224 } & \multicolumn{2}{|c|}{ IRAS 17245-3951 } & \multicolumn{2}{|c|}{ IRAS 17441-2411 } \\
\hline & $T_{\mathrm{mb}}$ & $\int T_{\mathrm{mb}} \mathrm{d} v$ & $T_{\mathrm{mb}}$ & $\int T_{\mathrm{mb}} \mathrm{d} v$ & $T_{\mathrm{mb}}$ & $T_{\mathrm{mb}} \mathrm{d} v$ & $T_{\mathrm{mb}}$ & $\int T_{\mathrm{mb}} \mathrm{d} v$ & $T_{\mathrm{mb}}$ & $\int T_{\mathrm{mb}} \mathrm{d} v$ & $T_{\mathrm{mb}}$ & $T_{\mathrm{mb}} \mathrm{d} v$ \\
\hline & $\mathrm{K}$ & $\mathrm{K} \mathrm{km} \mathrm{s}^{-1}$ & $\mathrm{~K}$ & $\mathrm{~K} \mathrm{~km} \mathrm{~s}^{-1}$ & K & $\mathrm{K} \mathrm{km} \mathrm{s}^{-1}$ & K & $\mathrm{K} \mathrm{km} \mathrm{s}^{-1}$ & K & $\mathrm{K} \mathrm{km} \mathrm{s}^{-1}$ & K & $\mathrm{K} \mathrm{km} \mathrm{s}^{-1}$ \\
\hline $\mathrm{HCN}(J=1-0)$ & - & $<0.07$ & 0.02 & 0.47 & - & - & 0.02 & 0.54 & - & $<0.09$ & 0.05 & 0.32 \\
\hline $\operatorname{CO}(J=2-1)$ & 0.09 & 2.07 & 1.75 & 32.60 & 0.30 & 1.84 & 0.65 & 12.84 & 0.04 & 0.58 & 0.57 & 6.24 \\
\hline$I_{\mathrm{HCN}} / I_{\mathrm{CO}}$ & & $<0.04$ & & 0.01 & & - & & 0.04 & & $<0.15$ & & 0.05 \\
\hline
\end{tabular}

Table 5. Adopted and derived modelling parameters of the two PPNe.

\begin{tabular}{|c|c|c|c|c|c|c|c|c|}
\hline IRAS No. & $\begin{array}{c}D \\
{[\mathrm{kpc}]}\end{array}$ & $\begin{array}{c}L \\
{\left[L_{\odot}\right]}\end{array}$ & $f_{\mathrm{CO}}$ & $\begin{array}{c}\dot{M} \\
{\left[M_{\odot} \mathrm{yr}^{-1}\right]}\end{array}$ & $\begin{array}{c}v_{\exp } \\
{\left[\mathrm{km} \mathrm{s}^{-1}\right]}\end{array}$ & $\begin{array}{c}R_{\mathrm{e}} \\
{[\mathrm{cm}]}\end{array}$ & $\bar{h}$ & ${ }^{12} \mathrm{CO} /{ }^{13} \mathrm{CO}$ \\
\hline $16594-4656$ & $1.80^{a}$ & 4900 & $1 \times 10^{-3}$ & $1 \times 10^{-5}$ & 14.0 & $2.9 \times 10^{17}$ & 10.0 & 30 \\
\hline $17150-3224$ & $2.42^{b}$ & 11000 & $2 \times 10^{-4}$ & $3 \times 10^{-5}$ & 14.5 & $2.4 \times 10^{17}$ & 1.8 & 7 \\
\hline
\end{tabular}

$\left(R_{\mathrm{e}}\right)$ and ${ }^{12} \mathrm{CO} /{ }^{13} \mathrm{CO}$-ratio are reported in Table 5 together with the adopted distance $(D)$, luminosity $(L)$ and initial CO fractional abundance $\left(f_{\mathrm{CO}}\right)$. Also shown is the $h$-parameter that determines the amount of heating in the envelope due to momentum transfer due to dust-gas collisions (see Schöier \& Olofsson 2001, for details).

In Fig. 6 the best fit model for IRAS 16594-4656, using a mass loss rate of $1 \times 10^{-5} M_{\odot} \mathrm{yr}^{-1}$ and a ${ }^{12} \mathrm{CO} /{ }^{13} \mathrm{CO}$-ratio of 30 , is overlayed on the observed spectra. The total integrated intensities in the lines are well reproduced as are the overall line profiles. There is confusion due to interstellar lines at the redshifted edge of the line profiles. Also, on the blue-shifted edge there are signs of a second weak component, possibly a higher velocity wind due to the present-day mass loss.

A good fit to $\mathrm{CO}$ observations is also obtained for IRAS $17150-3224$ using a mass loss rate of $3 \times 10^{-5} M_{\odot} \mathrm{yr}^{-1}$ (Fig. 7). The ${ }^{12} \mathrm{CO} /{ }^{13} \mathrm{CO}$-ratio is 7 , significantly lower than for IRAS 16594-4656 but consistent with IRAS 17150-3224 being O-rich (Abia et al. 2001). The ${ }^{12} \mathrm{CO}$ spectra for this source show signs of excess emission at larger expansion velocities possibly indicating the presence of a faster moving wind. However, the blue side of the emission is confused by interstellar line contamination.

The $h$-parameter of 10 derived for IRAS 16594-4656 is significantly larger than that of IRAS 17150-3224 and that which is typically derived for high mass-loss rate AGB-stars, $h \sim 1-2$, (Schöier \& Olofsson 2001; Woods et al. 2003b). The kinetic temperature derived through the envelope of IRAS 16594-4656 is about a factor of two, on average, larger than that obtained for the carbon star IRC+10216 (Schöier \& Olofsson 2000, 2001). Such a large discrepancy is hard to explain due to, e.g., uncertainties in the adopted distance, and could instead indicate that the properties of the dust grains are different in IRAS $16594-4656$ or that there is an additional source of heating of the envelope. A further possibility is that the spectra are contaminated by a second, warmer component such as the bipolar present day mass-loss seen in optical images at smaller spatial scales.
The winds of these PPNe are assumed to resemble those of their progenitor AGB stars, i.e., spherical symmetry and a constant expansion velocity are assumed. Given that both sources left the AGB 200-400 yr ago (see Sect. 7.3) this assumption should be valid for distances $\gtrsim 1 \times 10^{16} \mathrm{~cm}$, since the material outside this radius would once have made up the AGB wind. However, there is the possibility that a high velocity bipolar wind is penetrating this remnant AGB shell. The CO emission from the lower rotational transitions observed here are formed mostly at distances larger than $5 \times 10^{16} \mathrm{~cm}$ and thus mainly probe the outer, AGB-type, wind. For other molecular species (e.g., HCN) contributions from the present-day wind might become significant.

However, a more detailed treatment of the wind characteristics will require high-resolution, multi-transition observations that will become a reality with the upcoming APEX/ALMA telescopes. Deviations from the AGB wind model will become more apparent in the high- $J$ CO lines, both in the form of the line profile (broader wings) and in the intensity. The AGB wind model predicts that the higher-J CO lines $(J=4-3$ to $13-12)$ should all have an integrated intensity of about $30 \mathrm{~K} \mathrm{~km} \mathrm{~s}^{-1}$ and $12 \mathrm{~K} \mathrm{~km} \mathrm{~s}^{-1}$ for IRAS $16594-4656$ and IRAS 17150-3224, respectively, when observed with the APEX telescope. Also, the effect of dust emission in the excitation of the molecules, which is not taken into account in the present analysis, needs to be investigated.

\section{Molecular evolution}

\subsection{Molecules}

The molecules seen in PPNe give information on various processes which have occurred in the history of the particular PPN. $\mathrm{HCN}$ and $\mathrm{CN}$ can be used to give an idea of the evolutionary status of a PPN: in AGB stars, HCN is usually more abundant than $\mathrm{CN}$. As HCN becomes increasingly photodissociated, its daughter, $\mathrm{CN}$, becomes increasingly abundant; i.e., one would expect the $\mathrm{CN} / \mathrm{HCN}$ ratio to increase with evolutionary age, in the early post-AGB phase (see Fig. 8). A similar effect is seen 

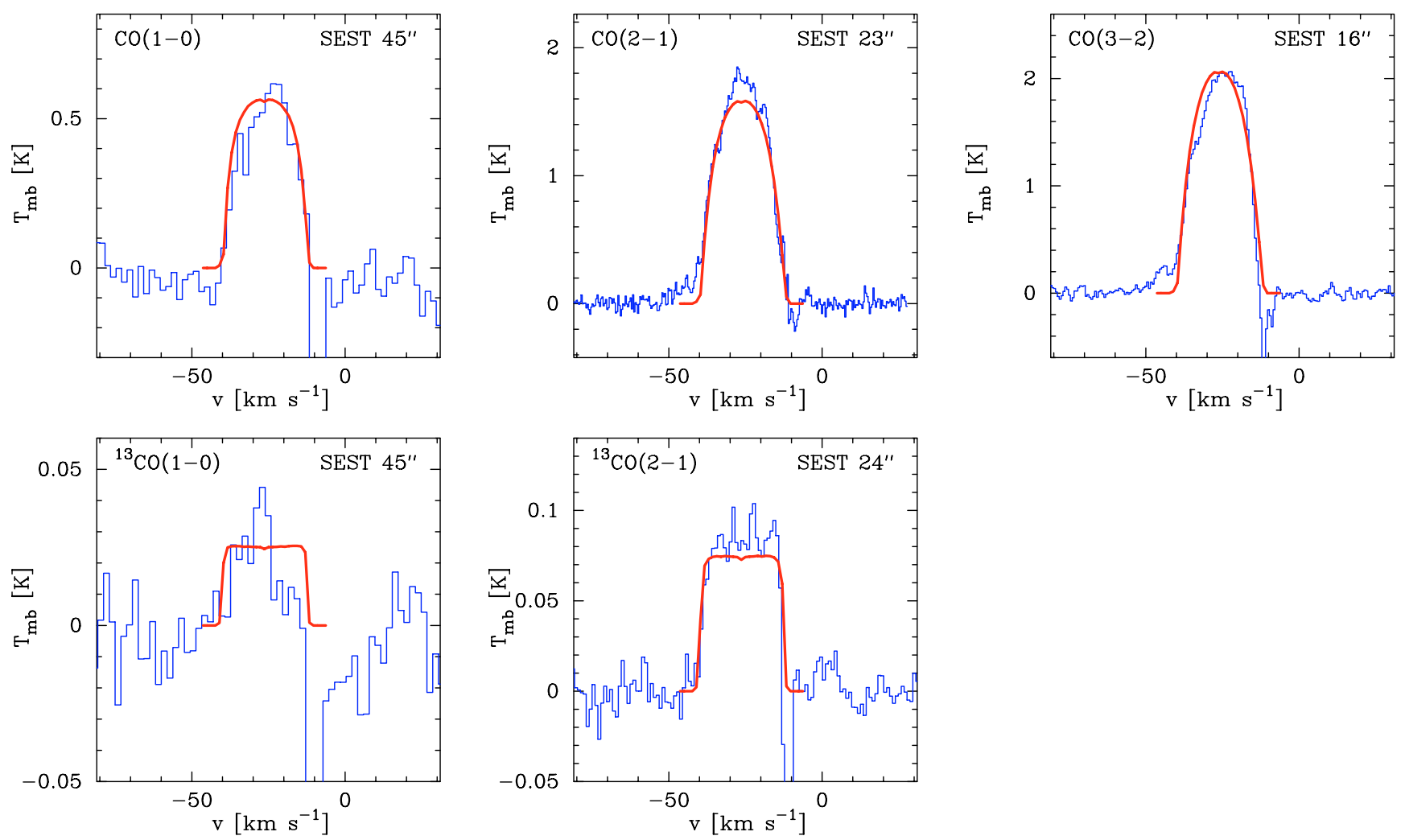

Fig. 6. Best fit CO model of IRAS 16594-4656 (solid line) overlayed on observations (histogram) using a mass loss rate of $1 \times 10^{-5} M_{\odot} \mathrm{yr}^{-1}$ and a ${ }^{12} \mathrm{CO} /{ }^{13} \mathrm{CO}$-ratio of 30 .
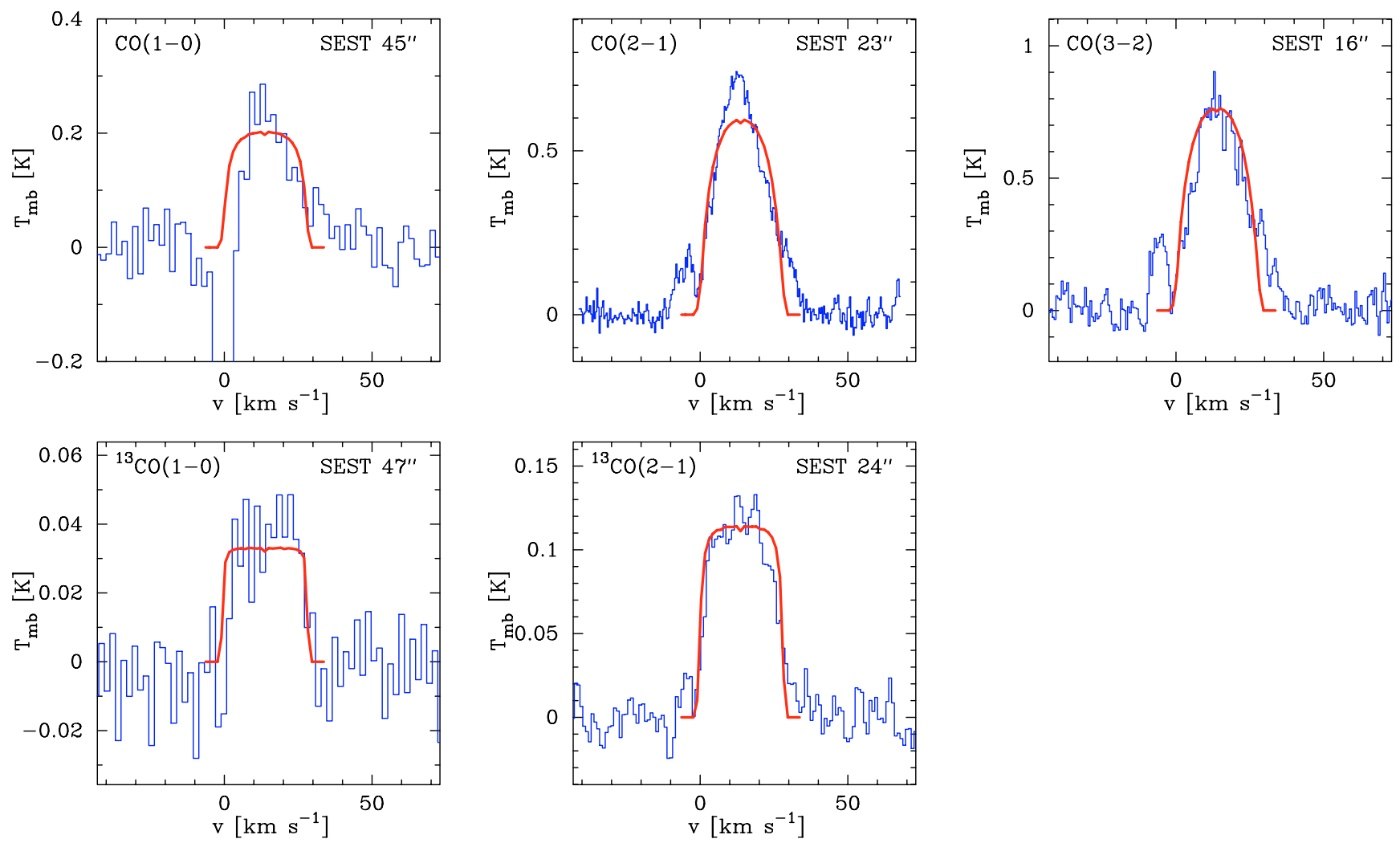

Fig. 7. Best fit CO model of IRAS 17150-3224 (solid line) overlayed on observations (histogram) using a mass loss rate of $3 \times 10^{-5} M_{\odot} \mathrm{yr}^{-1}$ and a ${ }^{12} \mathrm{CO} /{ }^{13} \mathrm{CO}$-ratio of 7 . 


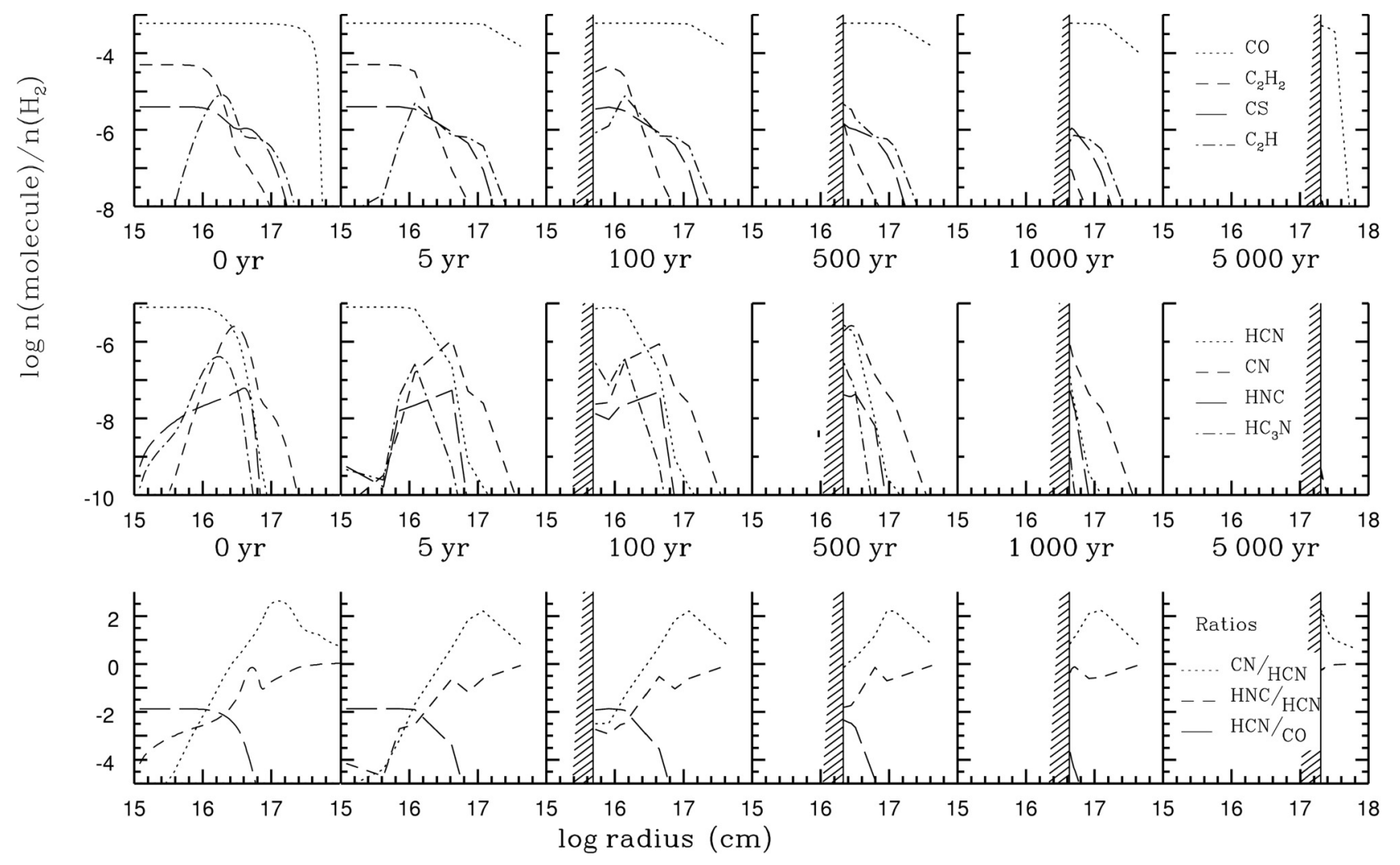

Fig. 8. Results of the chemical model of IRAS 16594-4656. The figure shows the variation of the fractional abundances (or ratios, in the lower row of axes) of selected species radially through the expanding circumstellar envelope at different points in time (measured in years since the cessation of mass-loss). The shading marks the passage of the inner edge of the circumstellar envelope. As can be seen at long times (5000 yr), only CO is reasonably abundant. The three sets of axes labelled " $0 \mathrm{yr}$ " show the results of a standard AGB model (see Sect. 6.3.2).

in the comparison of HCN and HNC: In CRL618, for example, the column densities of HCN and HNC are comparable (Herpin \& Cernicharo 2000), whereas in IRC+10216 (a less evolved object) $\mathrm{HCN}$ is far more abundant that HNC. In both these objects $\mathrm{HNC}$ is formed via dissociative recombination of $\mathrm{HCNH}^{+}$.

The HCN/CO ratio should also give some indication of age, since photodissociation would cause this ratio to decrease with time. However, it may be difficult to compare a number of objects which includes a mixture of O-rich and C-rich stars, since they may have had a different $\mathrm{HCN} / \mathrm{CO}$ ratio when they started to move off the AGB.

The presence of CS can be used in a similar way. CS is abundant in AGB envelopes, and although it can be enhanced by shocks in PPNe (Kasuga et al. 1997; Mitchell 1984) is generally of lower abundance, and unobserved in $\mathrm{PNe}$ until recently (Woods \& Nyman 2004). $\mathrm{HC}_{3} \mathrm{~N}, \mathrm{SiO}$ and $\mathrm{SiC}_{2}$ demonstrate the same sort of behaviour, decreasing in abundance going into the PPNe phase.

$\mathrm{HCO}^{+}$becomes greatly enhanced in the PPNe phase, and yet it is very difficult to explain why. Theoretical models of post-AGB objects all fail to produce enough $\mathrm{HCO}^{+}$by at least an order of magnitude, if not more (e.g., Howe et al. 1994; Ali et al. 2001; Woods et al. 2003a). $\mathrm{HCO}^{+}$is only efficiently formed by ionising radiation, and yet the levels of cosmic ray ionising radiation required to produce the desired levels of $\mathrm{HCO}^{+}$result in the destruction of most other molecules. This is due to the fact that cosmic rays induce UV photons in their interaction with $\mathrm{H}_{2}$; these photons dissociate and ionise molecules. Woods et al. (2003a) considered whether X-rays might be the responsible for the high level of $\mathrm{HCO}^{+}$. Their treatment was quite general - a treatment where X-rays are solely responsible for ionisation (and not dissociation) might prove interesting. This approach was taken in the chemical model explained in Sect. 6.3. However, a more thorough treatment needs to be made.

$\mathrm{HCO}^{+}$can also form in photon-dominated regions (PDRs), in warm gas $(800 \mathrm{~K})$, through reactions involving $\mathrm{CO}^{+}$, which itself is formed through reactions between $\mathrm{C}^{+}$and $\mathrm{OH}$ (Hasegawa et al. 2000). Moreover, shocked gas can be identified by the presence of $\mathrm{HCO}^{+}$(as well as $\mathrm{SiO}$ ) (e.g., Mitchell \& Watt 1985), and although high levels of $\mathrm{HCO}^{+}$do not necessarily imply shocks, the presence of high levels of $\mathrm{SiO}$ does give a much better indication of the presence of shocked gas.

The ${ }^{12} \mathrm{CO} /{ }^{13} \mathrm{CO}$ is low $(<10)$ in several PPNe (e.g., Kahane et al. 1992) compared to $10<{ }^{12} \mathrm{CO} /{ }^{13} \mathrm{CO}<25$ in evolved PNe (Palla et al. 2000; Bachiller et al. 1997a), $10<{ }^{12} \mathrm{CO} /{ }^{13} \mathrm{CO}<40$ in compact PNe (Josselin \& Bachiller 2003) and $10 \lesssim{ }^{12} \mathrm{CO} /{ }^{13} \mathrm{CO} \lesssim 50$ in AGB stars (e.g., Kahane et al. 1988; Greaves \& Holland 1997; Schöier \& Olofsson 2000). From the radiative transfer analysis ${ }^{12} \mathrm{CO} /{ }^{13} \mathrm{CO}$ ratios of 30 and 7 are derived for the PPNe IRAS 16594-4656 and 
Table 6. Calculated fractional abundances, with respect to $n\left(\mathrm{H}_{2}\right)$.

\begin{tabular}{|c|c|c|c|c|c|c|c|}
\hline \multirow[b]{2}{*}{ Molecule } & \multirow[b]{2}{*}{ Transition } & \multicolumn{3}{|c|}{ IRAS 16594-4656 } & \multicolumn{3}{|c|}{ IRAS 17150-3224 } \\
\hline & & $\begin{array}{c}r_{\mathrm{i}} \\
\mathrm{cm}\end{array}$ & $\begin{array}{c}r_{\mathrm{e}} \\
\mathrm{cm}\end{array}$ & $f_{\mathrm{X}}$ & $\begin{array}{c}r_{\mathrm{i}} \\
\mathrm{cm}\end{array}$ & $\begin{array}{c}r_{\mathrm{e}} \\
\mathrm{cm}\end{array}$ & $f_{\mathrm{X}}$ \\
\hline${ }^{13} \mathrm{CO}^{a}$ & - & - & $2.9(17)$ & $3.3(-5)$ & - & $2.4(17)$ & $2.9(-5)$ \\
\hline $\mathrm{HCN}$ & $J=1-0$ & - & $2.3(16)$ & $1.0(-6)$ & - & $4.4(16)$ & $3.8(-7)$ \\
\hline $\mathrm{HCN}$ & $J=3-2$ & - & $2.3(16)$ & $1.9(-7)$ & - & $4.4(16)$ & - \\
\hline $\mathrm{CN}$ & $N=1-0$ & $1.7(16)$ & $5.1(16)$ & $9.6(-6)$ & $3.9(16)$ & $9.8(16)$ & U.L. ${ }^{b}$ \\
\hline $\mathrm{CN}$ & $N=2-1$ & $1.7(16)$ & $5.1(16)$ & $2.5(-6)$ & $3.9(16)$ & $9.8(16)$ & U.L. ${ }^{b}$ \\
\hline
\end{tabular}

${ }^{a}{ }^{13} \mathrm{CO}$ fractional abundances derived from full radiative transfer (Sect. 5).

${ }^{b}$ Signifies a non-detection, but the calculation of an upper limit to the fractional abundance has been made (see Table 7).

Table 7. Calculated upper limits to fractional abundances, with respect to $n\left(\mathrm{H}_{2}\right)$.

\begin{tabular}{lccc}
\hline \hline & IRAS $16594-4656$ & IRAS 17150-3224 \\
\cline { 2 - 2 } Molecule & $f_{\mathrm{X}}$ & $f_{\mathrm{X}}$ \\
\hline $\mathrm{CO}^{+}$ & $1.1(-8)$ & - \\
$\mathrm{HCO}^{+}$ & $1.2(-7)$ & - \\
$\mathrm{H}_{2} \mathrm{CO}$ & $1.1(-6)$ & - \\
$\mathrm{CS}$ & $6.5(-8)$ & - \\
$\mathrm{C}_{2} \mathrm{~S}$ & $3.4(-7)$ & $9.5(-8)$ \\
$\mathrm{C}_{3} \mathrm{~S}$ & $4.1(-7)$ & - \\
$\mathrm{SiO}$ & $5.1(-8)$ & - \\
$\mathrm{SiS}$ & $3.2(-7)$ & - \\
$\mathrm{SiC}$ & $4.7(-7)$ & $2.1(-6)$ \\
$\mathrm{CN}$ & Det. & \\
${ }^{13} \mathrm{CN}$ & $5.0(-7)$ & $1.1(-8)$ \\
$\mathrm{H}^{13} \mathrm{CN}$ & $2.4(-8)$ & - \\
$\mathrm{HNC}^{13}$ & $1.8(-7)$ & $1.3(-8)$ \\
$\mathrm{HN}^{13} \mathrm{C}$ & $1.9(-7)$ & - \\
$\mathrm{HC}_{3} \mathrm{~N}$ & $2.7(-7)$ & - \\
$\mathrm{H}^{(13)} \mathrm{C}_{3} \mathrm{~N}^{b}$ & $2.7(-7)$ & - \\
$\mathrm{H}^{13} \mathrm{CCCN}$ & $2.0(-7)$ & - \\
$\mathrm{C}_{3} \mathrm{~N}$ & $4.2(-7)$ & - \\
$\mathrm{CH}_{3} \mathrm{CN}$ & $8.2(-8)$ & - \\
$\mathrm{C}_{2} \mathrm{H}$ & $3.1(-6)$ & $5.3(-8)$ \\
$\mathrm{CH}_{3} \mathrm{C}_{2} \mathrm{H}$ & $3.5(-6)$ & - \\
$\mathrm{C}_{3} \mathrm{H}_{2}$ & $2.5(-7)$ & - \\
$\mathrm{C}_{4} \mathrm{H}$ & $4.5(-6)$ & - \\
\hline
\end{tabular}

${ }^{a}$ Signifies a detection has been made of that particular line in that particular source (see Table 6).

${ }^{b}$ Signifies a blend of $\mathrm{HCC}^{13} \mathrm{CN}$ and $\mathrm{HC}^{13} \mathrm{CCN}$.

IRAS $17150-3224$, respectively. This ratio may be an indicator of evolution (since ${ }^{13} \mathrm{CO}$ self-shields less than ${ }^{12} \mathrm{CO}$ ), but it is more likely to be an indicator of a star's nucleosynthesis history which may be very different for otherwise apparently similar objects (e.g., Kahane et al. 1992, 1988).

\subsection{Fractional abundances}

\subsubsection{Calculation of fractional abundances}

In Sect. 5 the mass loss rates, and hence the radial distribution of the number density of $\mathrm{H}_{2}$, for the sample PPNe were determined from a detailed radiative transfer analysis of the observed $\mathrm{CO}$ line emission adopting a particular $\mathrm{CO} / \mathrm{H}_{2}$ abundance ratio. Here, fractional abundances for the remaining molecular species observed were calculated assuming optically thin emission. The radiative transfer for these lines is treated in the same way as described in Woods et al. (2003b), and the results are shown in Table 6. A constant excitation temperature of $25 \mathrm{~K}$ is assumed throughout the emitting region. Upper limits to fractional abundances are given in Table 7, and are calculated using the integrated intensity from Eq. (1). The same molecular data as used in Woods et al. (2003b) are adopted here.

Inner and outer radii of molecular distributions are determined in much the same way as Woods et al. (2003b), using a photodissociation model. Here, however, there is not the benefit of good interferometric data. Hence simplifying assumptions have to be made about certain molecular distributions. The distribution of all parent species, with the exception of $\mathrm{CO}$, is calculated from the photodissociation model (observed parent species are $\mathrm{HCN}, \mathrm{CS}, \mathrm{SiO}, \mathrm{SiS}$ ). The distribution of $\mathrm{CO}$ comes from the radiative transfer modelling, as described in Sect. 5. Self-shielding of CO is taken into account, in the manner of Mamon et al. (1988). All ionisation products or products of the circumstellar chemistry are assumed to take on the distribution of $\mathrm{C}_{2} \mathrm{H}$, with the exception of $\mathrm{CN}$, which has a slightly more expansive distribution. This should not have too great an effect on results since there is only a linear dependence on the difference between inner and outer radius. These are very straightforward assumptions, which ignore effects of circumstellar chemistry, and freeze-out onto grains, for example. Again, isotopomers are assumed to have the same distribution as their more abundant forms.

\subsubsection{Uncertainties}

As discussed in Woods et al. (2003b), there are inherent uncertainties in the approach taken here. Typical distance estimates can vary by up to a factor 2 , and this influences the mass-loss rate derived from the radiative transfer modelling (see Sect. 5). When combined with the uncertainties in the choice of inner and outer radii for the molecular distributions, the overall error in fractional abundance varies with $\sim D^{-1-0}$, where $D$ is the distance. Given also that there are likely to be errors introduced by the choice of excitation temperature, an overall error of a factor 5 or so is to be expected in fractional abundance 
estimates. Moreover, some species other than $\mathrm{CO}$ are expected to be optically thick, most notably $\mathrm{HCN}$, and the optically thin approximation would give fractional abundances which are systematically too low in those cases. As mentioned in Sect. 5, contribution to the line intensities from the present-day, nonspherically symmetric, high-velocity, mass loss might become significant for some molecules. A full treatment of this problem, including the effects of radiative excitation due to dust emission, will require high spatial resolution observations of a large number of molecules. With these caveats, molecular fractional abundances are believed to be order of magnitude estimates.

\subsection{The chemical model}

To investigate the changes in fractional abundance of species during the late AGB and PPN phases, a chemical model of the circumstellar envelope was used. Results of this model are shown in Fig. 8. The chemical model of IRC +10216 constructed by Millar et al. (2000, MHB) has been adapted to investigate the chemistry, particularly the photochemistry, as an AGB star finishes its phase of mass loss, and moves into the PPN phase. Several simplifying assumptions are made, and the MHB model has been changed to include X-rays, and a minimal X-ray chemistry.

\subsubsection{Model parameters and simplifying assumptions}

Several parcels of gas and dust in the circumstellar envelope (CSE) of an AGB star are assumed to flow outward at a uniform velocity of $14 \mathrm{~km} \mathrm{~s}^{-1}$, from initial radial distances of 1,4 and $7 \times 10^{15}, 1,4$ and $7 \times 10^{16}$ and 1 and $4 \times 10^{17} \mathrm{~cm}$ from the central star. A mass-loss rate of $1 \times 10^{-5} M_{\odot} \mathrm{yr}^{-1}$ is chosen, in accordance with the molecular line modelling in Table 5. A spherical geometry implies that the density of the homogeneous outflow drops as $r^{-2}$. Mass-loss is assumed to end with the start of the model.

Envelope heating. During the expansion, the "kinetic temperature" of the parcels varies according to $T=T_{0}\left(r_{0} / r\right)^{0.79}$, where $T_{0}$ increases from $100 \mathrm{~K}$ to $1000 \mathrm{~K}$ over the first $1000 \mathrm{yr}$ of the model (chosen to represent the PPN phase). This heating is due to the increasing temperature of the central star, as it evolves towards a white dwarf.

Stellar UV field. Following the increase in stellar temperature, the intensity of the stellar UV field also increases. An ionisation rate similar to that of the interstellar UV field is assumed at the start of the model. At the end of the first $1000 \mathrm{yr}$ this value has increased by a factor of $2 \times 10^{5}$ (cf., Herpin \& Cernicharo 2000).

$X$-rays. X-rays from the central star or companion (Woods et al. 2002) are treated in a very simple way in this model. The X-ray extinction law of Deguchi et al. (1990) is used (see their Eq. (B3)), and an X-ray energy of $1.2 \mathrm{keV}$ is assumed $(\lambda \sim 1 \mathrm{~nm})$. X-rays only play an ionising role in the chemical reaction network, and these reactions are given the same rate coefficients as the equivalent cosmic-ray reactions. A very low ionisation rate of $10^{-17} \mathrm{~s}^{-1}$ is chosen initially; this has risen to a rate of $10^{-13} \mathrm{~s}^{-1}$ by the end of the model run.

\subsubsection{Chemical reaction network}

The reaction network is the same as that used in Woods et al. (2003a), with the addition of a small number of X-ray ionisation reactions. At present the network includes 3897 reactions among 407 species in 6 elements. Initial fractional abundances are determined via the use of an AGB steady-state model, with a mass-loss rate of $1 \times 10^{-5} M_{\odot} \mathrm{yr}^{-1}$ and expansion velocity of $14 \mathrm{~km} \mathrm{~s}^{-1}$. For example, a parcel starting at a radius of $1 \times 10^{16} \mathrm{~cm}$ in the model presented here will have initial abundances which are the same as a parcel of "AGB" material at the same radial distance. The initial abundances of parent molecules for the AGB model are chosen to represent a carbonrich environment, and values are taken from MHB.

\section{Discussion}

\subsection{Comparison of observations with chemical model}

The chemical model generally shows good agreement with the fractional abundances derived here. Given the order of magnitude error margins (Sect. 6.2.2), both $\mathrm{HCN}$ and $\mathrm{CN}$ reach the calculated fractional abundances for IRAS 16594-4656 during the first $1000 \mathrm{yr}$ of the model (i.e., the PPN phase). Upper limits for other observed species (e.g., $\mathrm{HNC}, \mathrm{HC}_{3} \mathrm{~N}, \mathrm{C}_{2} \mathrm{H}$ and $\mathrm{C}_{4} \mathrm{H}$ ) are also in agreement with the model in this region. $\mathrm{CS}$ is predicted to be more abundant than observed. The model also shows that a few species (e.g., $\mathrm{CO}, \mathrm{C}_{2} \mathrm{H}$ and $\mathrm{CN}$ ) survive at observable levels after, say, $1000 \mathrm{yr}$ of post-AGB evolution (Fig. 8).

Perhaps the most interesting result of the model is the variation of the $\mathrm{CN} / \mathrm{HCN}, \mathrm{HNC} / \mathrm{HCN}$ and $\mathrm{HCN} / \mathrm{CO}$ ratios. As expected, the $\mathrm{CN} / \mathrm{HCN}$ ratio is seen to rise during the early postAGB evolution (taking a mean through the envelope, Fig. 8). Similarly, the HNC/HCN ratio also increases. The HCN/CO ratio declines significantly with post-AGB age. When these three behaviours are combined, a reasonably accurate age can be given. This approach is taken later, in Sect. 7.3.

\subsection{Comparison of observations with other observations}

IRAS 16594-4656 is very under-abundant in molecules in comparison to C-rich AGB stars. Generally, fractional abundances derived here, and presented in Table 6 (and the upper limits shown in Table 7), are an order of magnitude deficient when compared to C-rich AGB stars, such as those surveyed in Bujarrabal et al. (1994) and Woods et al. (2003b). Two exceptions to this are $\mathrm{CN}$ and ${ }^{13} \mathrm{CO}$, which show good agreement with the fractional abundances derived for IRC +10216 .

The lack of detection of $\mathrm{SiO}$ would seem to confirm that IRAS 16954-4656 is C-rich, and that any silicate grains present (see García-Lario et al. 1999) are not being destroyed by shocks because either shocks might not be present, or the shocks which are present are not strong enough (cf., van de Steene \& van Hoof 2003). Another explanation may be that the shocked regions are very small, in which case high abundances of $\mathrm{SiO}$ would be localised, and $\mathrm{SiO}$ emission diluted by the beam. 
Table 8. Comparison with fractional abundances in other PPNe, arranged in order of decreasing stellar temperature.

\begin{tabular}{|c|c|c|c|c|c|c|}
\hline C-rich PPNe: & IRAS $16594-4656$ & M1-16 & CRL618 & CRL2688 & IRAS 19500-1709 & IRC+10216 \\
\hline${ }^{13} \mathrm{CO}$ & $3.3(-5)^{a}$ & & $2.4(-5)^{b}$ & $5.2(-5)^{b}$ & $2.0(-5)^{k}$ & \\
\hline $\mathrm{HCN}$ & $6.1(-7)^{a}$ & $1.0(-7)^{j}$ & $4.4(-6)^{b}-5.0(-7)^{d}$ & $2.1(-5)^{b}-4.0(-7)^{d}$ & $1.5(-7)^{k}$ & $1.3(-5)^{r}$ \\
\hline $\mathrm{CN}$ & $6.0(-6)^{a}$ & $8.5(-7)^{j}$ & $2.1(-6)^{c}$ & $1.0(-6)^{c}$ & & $2.2(-6)^{r}$ \\
\hline $\mathrm{H}^{13} \mathrm{CN}$ & $<2.4(-8)^{a}$ & & $6.3(-8)^{e}$ & $>4.0(-6)^{g}-5.1(-7)^{e}$ & & $2.8(-7)^{r}$ \\
\hline $\mathrm{HNC}$ & $<1.8(-7)^{a}$ & & $1.9(-6)^{b}-1.9(-7)^{e}$ & $1.8(-7)^{h}-5.0(-8)^{g}$ & & $5.5(-8)^{r}$ \\
\hline $\mathrm{HC}_{3} \mathrm{~N}$ & $<2.7(-7)^{a}$ & & $4.4(-7)^{e}-1.9(-7)^{b}$ & $1.5(-7)^{e, g}$ & & $1.0(-6)^{r}$ \\
\hline $\mathrm{HCO}^{+}$ & $<1.2(-7)^{a}$ & $9.3(-8)^{j}$ & $2.0(-7)^{d}$ & & & \\
\hline CS & $<6.5(-8)^{a}$ & $<8.8(-8)^{j}$ & $4.1(-7)^{b}-6.0(-8)^{d}$ & $2.1(-6)^{b}$ & & $8.8(-7)^{r}$ \\
\hline $\mathrm{C}_{2} \mathrm{H}$ & $<3.1(-6)^{a}$ & & $2.0(-6)^{d}$ & $9.3(-6)^{f}$ & & $2.6(-6)^{r}$ \\
\hline $\mathrm{C}_{4} \mathrm{H}$ & $<4.5(-5)^{a}$ & & $2.9(-6)^{b}-8.0(-8)^{d}$ & & & $3.2(-6)^{r}$ \\
\hline $\mathrm{SiO}$ & $<5.1(-8)^{a}$ & $<2.4(-8)^{j}$ & $<5.5(-7)^{b}$ & & & $1.1(-7)^{r}$ \\
\hline $\mathrm{SiS}$ & $<3.2(-7)^{a}$ & $<4.2(-8)^{j}$ & & $4.0(-8)^{g}$ & & $9.5(-7)^{r}$ \\
\hline O-rich PPNe: & IRAS 17150-3224 & M1-92 & HD101584 & IRAS 19114+0002 & OH17.7-2.0 & $\mathrm{OH} 231.8+4.2$ \\
\hline${ }^{13} \mathrm{CO}$ & $2.9(-5)^{a}$ & & $5.0(-4)^{q}$ & $3.8(-4)^{b}$ & $4.2(-6)^{b}$ & $1.0(-4)^{l}-2.2(-5)^{b}$ \\
\hline $\mathrm{HCN}$ & $3.8(-7)^{a}$ & $<2.0(-7)^{o, p}$ & $<1.7(-6)^{q}$ & $<4.5(-6)^{b}$ & $<1.6(-7)^{b}$ & $4.4(-6)^{b}-5.2(-8)^{m}$ \\
\hline $\mathrm{CN}$ & $<1.1(-8)^{a}$ & & & & & $<2.0(-7)^{c, l}$ \\
\hline $\mathrm{H}^{13} \mathrm{CN}$ & $<1.3(-8)^{a}$ & & & & & $4.8(-8)^{l}$ \\
\hline $\mathrm{C}_{4} \mathrm{H}$ & $<1.4(-6)^{a}$ & $<3.6(-7)^{q}$ & & $<1.4(-6)^{b}$ & $<4.7(-8)^{b}$ & \\
\hline
\end{tabular}

${ }^{a}$ This work, ${ }^{b}$ Bujarrabal et al. (1994), ${ }^{c}$ Bachiller et al. (1997b), ${ }^{d}$ Bujarrabal et al. (1988), ${ }^{e}$ Sopka et al. (1989), ${ }^{f}$ Fuente et al. (1998), ${ }^{g}$ Nguyen-Q-Rieu \& Bieging (1990), ${ }^{h}$ Kasuga et al. (1997), ${ }^{j}$ Sahai et al. (1994), ${ }^{k}$ Using the results of Bujarrabal et al. (1992, 2001), ${ }^{l}$ Morris et al. (1987), ${ }^{m}$ Sanchez Contreras et al. (1997), ${ }^{n}$ Omont et al. (1993), ${ }^{o}$ Lindqvist et al. (1992), ${ }^{p}$ Nercessian et al. (1989), ${ }^{q}$ Using the results of Olofsson \& Nyman (1999), ${ }^{r}$ Woods et al. (2003b).

Conversely, IRAS 17150-3224 seems (chemically) very much like an O-rich AGB star, such as those observed by Bujarrabal et al. (1994), or Lindqvist et al. (1988), for example. Although the fractional abundances of only two molecules, ${ }^{13} \mathrm{CO}$ and $\mathrm{HCN}$, in IRAS $17150-3224$ are suitable for comparison, they are very similar to the O-rich AGB stars in both AGB-star samples.

So, IRAS 16594-4656 and IRAS 17150-3224 seem to have different chemical properties from each other, and both seem far from the well-known molecule-rich PPNe, like CRL618, CRL2688 and OH231.8+4.2. IRAS 16594-4656, supposedly a C-rich object, is poor in molecules and, as can be seen from Table 8, is at least an order of magnitude less abundant than CRL618 and CRL2688 in many molecules. However, IRAS 16594-4656 has generally higher fractional abundances than M1-16, which is widely accepted to be a standard PN. An exception to this is $\mathrm{CN}-$ a fractional abundance of $\mathrm{CN}$ similar to those in CRL618 and CRL2688 may indicate that IRAS $16594-4656$ is a somewhat evolved PPN, but not quite part of the PN regime yet. The theoretical work of Woods et al. (2003a) shows that fractional abundances of $\mathrm{CN}$ can remain high for a period of $\sim 100$ years in the PPNe phase, whilst other molecules are destroyed, if molecular material is to be found in the shielded environment of a circumstellar torus.

Bachiller et al. (1997a) present a homogeneous sample of molecular abundances in post-AGB objects; however, both IRAS 16594-4656 and IRAS 17150-3224 are too low in molecular abundance to present much of a comparison. These two PPNe are closest in fractional abundances to the younger objects in the Bachiller et al. (1997a) sample.

Using the fractional abundance of HCN derived from the $J=1-0$ line, since this line is least likely of the two observed to be optically thick, a $\mathrm{CN} / \mathrm{HCN}$ ratio of $<10$ is derived for IRAS 16594-4656. This value is quite uncertain, and dependent very much on the factors discussed in Sect. 6.2.2. A full radiative transfer treatment of the $\mathrm{HCN}(J=1-0)$ line does in fact predict a fractional abundance 2-3 times larger than that derived via the optically thin assumption (Sect. 6.2.2), implying that IRAS $16594-4656$ could have a $\mathrm{CN} / \mathrm{HCN}$ ratio as low as $\sim 3-5$. In this case the $\mathrm{CN} / \mathrm{HCN}$ ratio seems to indicate that IRAS 16594-4656 is just starting the post-AGB phase. Other PPNe have a $\mathrm{CN} / \mathrm{HCN}$ ratio up to an order of magnitude smaller, e.g., for CRL618, CN/HCN $~ 0.5$ and for CRL2688, $\mathrm{CN} / \mathrm{HCN} \sim 0.2$. IRC +10216 has a smaller $\mathrm{CN} / \mathrm{HCN}$ ratio yet, and the more evolved object NGC7027 has a $\mathrm{CN} / \mathrm{HCN}$ ratio of about 10.

Both IRAS 16594-4656 and IRAS 17150-3224 have very low HCN/CO ratios compared to CRL618, CRL2688 and $\mathrm{OH} 231.8+4.2$. IRAS $16594-4656$ has an $\mathrm{HCN} / \mathrm{CO}$ ratio of $6 \times 10^{-4}$, whereas CRL618 and CRL2688 have ratios of $6 \times 10^{-3}$ and $3 \times 10^{-2}$ respectively (Bujarrabal et al. 1994). IRAS $17150-3224$ has an $\mathrm{HCN} / \mathrm{CO}$ ratio of $2 \times 10^{-3}$, which is again smaller than the $2 \times 10^{-2}$ of $\mathrm{OH} 231.8+4.2$ (Bujarrabal et al. 1994).

From what can be implied by Table 8, IRAS $17150-3224$ is similar to $\mathrm{OH} 231.8+4.2$ in ${ }^{13} \mathrm{CO}$, and $\mathrm{HCN}$ (despite the spread in observed values). The relatively high fractional abundance of 
HCN (half that of the C-rich object IRAS 16594-4656) would imply that IRAS $17150-3224$ is at a stage before heavy ionisation of the molecular matter occurs (and the $\mathrm{CN}$ fractional abundance rises), i.e., IRAS 17150-3224 is at a less evolved stage than IRAS 16594-4656.

\subsection{Chemical evolution from the AGB}

One has to ask why there seem to be two differing evolutionary paths for PPNe: why are some PPNe moleculerich, like CRL618, and why are some molecule-poor, like the two objects discussed here? Visually, IRAS 16594-4656 and IRAS 17150-3224 are not very different from CRL618. All three have a similar bipolar structure, with a pinched waist indicating an equatorial density enhancement and possibly a collimating mechanism. In the case of CRL618, this mechanism could very well be a circumstellar torus, the presence of which has been implied from observations (see Herpin \& Cernicharo 2000, and references therein). Hence it does not seem too exotic to consider similar structures for IRAS 16594-4656 and IRAS 17150-3224. Many of the PPNe identified do have such structures, which have been directly observed (e.g., IRAS 17106-3046 - Kwok et al. 2000; IRAS 17245-3951 Hrivnak et al. 1999; IRAS 04296+3429 - Sahai 1999; Hen 401 - Sahai et al. 1999; IRAS 17441-2411 - Su et al. 1998; Kwok et al. 1996) and there is strong evidence from polarimetry ( $\mathrm{Su}$ et al. 2000) that a circumstellar disk or torus does exist around IRAS 16594-4656.

CRL618 owes its molecular richness, in a large part, to the shielding effects of its torus. This can clearly be seen in the chemical model of Woods et al. (2003a) - the high levels of radiation are only abated by high initial densities and a slow torus expansion velocity, and when the density, or essentially the extinction, becomes too low, complex molecules are destroyed. One obvious implication that this has for PPNe such as IRAS 16594-4656 and IRAS 17150-3224 is that their collimating torus must not be dense enough to synthesise and protect complex molecules. This brings in an interesting interplay between the column density of material required to produce the degree of collimation seen and the column density of material required to shield complex molecules from the often very intense UV fields, cosmic rays and possibly also X-rays. One of the prevalent results from Woods et al. (2003a) was that incident UV radiation would start to destroy molecules when the optical depth reached approximately 10 mag of extinction. This equates to a column density of $\sim 1.6 \times 10^{22} \mathrm{~cm}^{-2}$. For the particular model of CRL618, the density at the innermost part of the torus is $\sim 10^{9} \mathrm{~cm}^{-3}$. Numerical models (e.g., Ignace et al. 1996) show that only an equatorial density enhancement of 2 or more over the polar direction is enough to produce asphericity (bipolarity) in a wind-compressed disk situation. Typically an average AGB star (e.g., $\dot{M}=10^{-5} M_{\odot} \mathrm{yr}^{-1}$ and $v_{\exp }=15 \mathrm{~km} \mathrm{~s}^{-1}$ ) would have a density of around $10^{7} \mathrm{~cm}^{-3}$ at a similar radial distance $\left(10^{15} \mathrm{~cm}\right)$.

To support the hypothesis that these two PPNe are low in fractional abundances due to low envelope density, a chemical model was constructed to follow the evolution of a star along the AGB phase and into the PPNe phase. Details of the model can be found in Sect. 6.3. The model shows that within $\sim 500 \mathrm{yr}$ of the cessation of mass-loss UV photons from the star start to ionise the molecular material at a very high rate, and molecular matter is destroyed rapidly. Evidence of the increasing UV field can be seen particularly in the profiles of CN (Fig. 8), which show an increase in fractional abundance at the $500 \mathrm{yr}$ mark, where the UV field starts to become significant. The profiles shown in Fig. 8 are also subtly different from those of steadystate AGB models (compare with the leftmost set of axes, labelled " $0 \mathrm{yr}$ "), indicating that the mildly increasing UV field does have a marked effect, even in the early stages of postAGB evolution. X-ray photons have little effect in this environment, with the chosen assumptions. $\mathrm{CO}$, which self-shields in the model, would survive at least $12000 \mathrm{yr}$ after the end of mass-loss at detectable levels. This differs from the case of a molecular torus (Woods et al. 2003a) in that in the CSE case there is a reasonably slow decline of parent species, like HCN, whereas in the torus model, parent molecules are destroyed within a hundred years once the extinction provided by the circumstellar matter drops to around 10 magnitudes. It is also quite marked how rapidly species form in the torus before the "radiation catastrophe", in comparison with the slow build-up in the CSE model. As a consequence of this, there are not the high fractional abundances of photodissociation products (e.g., $\left.\mathrm{CN}, \mathrm{C}_{2} \mathrm{H}\right)$ in the CSE case that are found in the torus model, and in molecule-rich PPNe, like CRL618.

Using the fractional abundances calculated from observations and the chemical model an approximate post-AGB age for IRAS 16594-4656 can be estimated. IRAS 16594-4656 seems to be older than IRAS 17150-3224, with quite a high CN fractional abundance and also a higher $\mathrm{CN} / \mathrm{HCN}$ ratio. Through comparison with Fig. 8 in particular, the value of $370 \mathrm{yr}$, named by van der Veen et al. (1989), seems a good estimate, since a $\mathrm{CN} / \mathrm{HCN}$ ratio of $3-5$ (or even 10 ) is only possible at times less than 500 yr. IRAS $17150-3224$ is slightly more difficult to quantify, since there is no detection of CN. However, this does suggest that the $\mathrm{CN} / \mathrm{HCN}$ ratio is low. The $\mathrm{HCN} / \mathrm{CO}$ is high (higher than IRAS 16594-4656) and hence a post-AGB age of 150-210yr for IRAS 17150-3224 (Hu et al. 1993; Meixner et al. 2002) seems likely, with the estimate of van der Veen et al. (1989), $800 \mathrm{yr}$, appearing to be much too long to be an accurate post-AGB age for this object.

It is not clear whether IRAS 16594-4656 and IRAS 17150-3224 are older or younger than CRL618 and CRL2688. The lack of signs of ionisation suggest that they are younger; the reasonably high $\mathrm{CN} / \mathrm{HCN}$ ratios and low $\mathrm{HCN} / \mathrm{CO}$ ratios suggest that they are older. Certainly, IRAS 16594-4656 fits into the "evolutionary sequence" (of post-AGB objects) of Bachiller et al. (1997a) between CRL618 and the young PN, NGC7027. The age estimates of van der Veen et al. (1989), Hu et al. (1993) and Meixner et al. (2002), above, in comparison with the results of Bujarrabal et al. (2001) also tend to favour IRAS 16594-4656 and IRAS 17150-3224 being older, since Bujarrabal et al. (2001) give post-AGB ages for CRL618 and CRL2688 as 110 and $200 \mathrm{yr}$, respectively. 


\section{Conclusions}

Two proto-planetary nebulae, IRAS 16594-4656 and IRAS 17150-3224, were observed in a wide range of molecular lines, but only detected in a few. Calculating fractional abundances and upper limits from these lines shows that these two sources are molecule-poor in relation to other PPNe, such as CRL618, CRL2688 and OH231.8+4.2. As a reason for this apparent difference the degree of density of the circumstellar torus (or disk) is suggested, with molecule-rich PPNe having dense, protective and nurturing tori, and molecule-poor PPNe having tenuous, or no, tori. To substantiate this, a model of the chemistry in a late-AGB/early-PPN circumstellar envelope, with no particular density enhancements (such as a torus), is used. As expected, it shows that very few molecules reach high fractional abundances in the post-AGB phase, and agrees well with fractional abundances calculated from observations. This is in stark contrast to the model of Woods et al. (2003a), who model a dense circumstellar torus in the PPN phase. The usefulness of $\mathrm{HCN} / \mathrm{CO}, \mathrm{HNC} / \mathrm{HCN}$ and particularly $\mathrm{CN} / \mathrm{HCN}$ ratios in determining evolutionary age is discussed, and using these tools, post-AGB ages for IRAS 16594-4656 and IRAS 17150-3224 are given.

Acknowledgements. Astrophysics at UMIST is supported by a grant from PPARC. The Swedish-ESO Submillimetre Telescope, SEST, is operated jointly by ESO and the Swedish National Facility for Radioastronomy, Onsala Space Observatory at Chalmers University of Technology. F.L.S. and H.O. acknowledge financial support from the Swedish research council.

\section{References}

Abia, C., Busso, M., Gallino, R., et al. 2001, ApJ, 559, 1117

Ali, A., Shalabiea, O. M., El-Nawawy, M. S., \& Millar, T. J. 2001, MNRAS, 325, 881

Bachiller, R., Forveille, T., Huggins, P. J., \& Cox, P. 1997a, A\&A, 324, 1123

Bachiller, R., Fuente, A., Bujarrabal, V., et al. 1997b, A\&A, 319, 235

Balick, B. 1987, AJ, 94, 671

Balick, B., \& Frank, A. 2002, ARA\&A, 40, 439

Bujarrabal, V., Gomez-Gonzalez, J., Bachiller, R., \& Martin-Pintado, J. 1988, A\&A, 204, 242

Bujarrabal, V., Alcolea, J., \& Planesas, P. 1992, A\&A, 257, 701

Bujarrabal, V., Fuente, A., \& Omont, A. 1994, A\&A, 285, 247

Bujarrabal, V., Castro-Carrizo, A., Alcolea, J., \& Sánchez Contreras, C. 2001, A\&A, 377,868

Calvet, N., \& Peimbert, M. 1983, Rev. Mex. Astron. Astrofis., 5, 319

Cohen, M., \& Kuhi, L. V. 1977, ApJ, 213, 79

Deguchi, S., Izumiura, H., Kaifu, N., et al. 1990, ApJ, 351, 522

Fuente, A., Cernicharo, J., \& Omont, A. 1998, A\&A, 330, 232

García-Hernández, D. A., Manchado, A., García-Lario, P., et al. 2002, A\&A, 387, 955

García-Lario, P., Manchado, A., Ulla, A., \& Manteiga, M. 1999, ApJ, 513, 941

Greaves, J. S., \& Holland, W. S. 1997, A\&A, 327, 342

Hasegawa, T., Volk, K., \& Kwok, S. 2000, ApJ, 532, 994

Herpin, F., \& Cernicharo, J. 2000, ApJ, 530, L129

Howe, D. A., Hartquist, T. W., \& Williams, D. A. 1994, MNRAS, 271, 811

Hrivnak, B. J., Kwok, S., \& Su, K. Y. L. 1999, ApJ, 524, 849
Hrivnak, B. J., Volk, K., \& Kwok, S. 2000, ApJ, 535, 275

Hrivnak, B. J., Kwok, S., \& Su, K. Y. L. 2001, AJ, 121, 2775

Hu, J. Y., Slijkhuis, S., Nguyen-Q-Rieu, \& de Jong, T. 1993, A\&A, 273,185

Hu, J. Y., te Lintel Hekkert, P., Slijkhuis, F., et al. 1994, A\&AS, 103, 301

Ignace, R., Cassinelli, J. P., \& Bjorkman, J. E. 1996, ApJ, 459, 671

Josselin, E., \& Bachiller, R. 2003, A\&A, 397, 659

Kahane, C., Gomez-Gonzalez, J., Cernicharo, J., \& Guelin, M. 1988, A\&A, 190, 167

Kahane, C., Cernicharo, J., Gomez-Gonzalez, J., \& Guelin, M. 1992, A\&A, 256, 235

Kastner, J. H., \& Weintraub, D. A. 1994, ApJ, 434, 719

Kasuga, T., Yamamura, I., \& Deguchi, S. 1997, A\&A, 320, 575

Kwok, S. 1993, ARA\&A, 31, 63

Kwok, S., \& Hrivnak, B. J. 1989, in Infrared Spectroscopy in Astronomy, 363

Kwok, S., Hrivnak, B. J., Zhang, C. Y., \& Langill, P. L. 1996, ApJ, 472, 287

Kwok, S., Su, K. Y. L., \& Hrivnak, B. J. 1998, ApJ, 501, L117

Kwok, S., Hrivnak, B. J., \& Su, K. Y. L. 2000, ApJ, 544, L149

Lindqvist, M., Nyman, L.-A., Olofsson, H., \& Winnberg, A. 1988, A\&A, 205, L15

Lindqvist, M., Olofsson, H., Winnberg, A., \& Nyman, L.-A. 1992, A\&A, 263, 183

Loup, C., Forveille, T., Omont, A., \& Nyman, L.-A. 1990, A\&A, 227, L29

Mamon, G. A., Glassgold, A. E., \& Huggins, P. J. 1988, ApJ, 328, 797

Matsuura, M., Zijlstra, A. A., Molster, F. J., et al. 2004, ApJ, 604, 791

Meixner, M., Ueta, T., Bobrowsky, M., \& Speck, A. 2002, ApJ, 571, 936

Millar, T. J., Herbst, E., \& Bettens, R. P. A. 2000, MNRAS, 316, 195

Mitchell, G. F. 1984, ApJ, 287, 665

Mitchell, G. F., \& Watt, G. D. 1985, A\&A, 151, 121

Morris, M., Guilloteau, S., Lucas, R., \& Omont, A. 1987, ApJ, 321, 888

Nercessian, E., Omont, A., Benayoun, J. J., \& Guilloteau, S. 1989, A\&A, 210, 225

Nguyen-Q-Rieu, \& Bieging, J. H. 1990, ApJ, 359, 131

Nyman, L.-A., Hall, P. J., \& Olofsson, H. 1998, A\&AS, 127, 185

Olivier, E. A., Whitelock, P., \& Marang, F. 2001, MNRAS, 326, 490

Olnon, F. M., Raimond, E., Neugebauer, G., et al. 1986, A\&AS, 65, 607

Olofsson, H., \& Nyman, L.-Å. 1999, A\&A, 347, 194

Olofsson, H., Eriksson, K., \& Gustafsson, B. 1990, A\&A, 230, 405

Omont, A., Lucas, R., Morris, M., \& Guilloteau, S. 1993, A\&A, 267, 490

Palla, F., Bachiller, R., Stanghellini, L., Tosi, M., \& Galli, D. 2000, A\&A, 355, 69

Sahai, R. 1999, ApJ, 524, L125

Sahai, R., Wootten, A., Schwarz, H. E., \& Wild, W. 1994, ApJ, 428, 237

Sahai, R., Hines, D. C., Kastner, J. H., et al. 1998, ApJ, 492, L163

Sahai, R., Bujarrabal, V., \& Zijlstra, A. 1999, ApJ, 518, L115

Sanchez Contreras, C., Bujarrabal, V., \& Alcolea, J. 1997, A\&A, 327, 689

Schöier, F. L., \& Olofsson, H. 2000, A\&A, 359, 586

Schöier, F. L., \& Olofsson, H. 2001, A\&A, 368, 969

Schmidt, G. D., Hines, D. C., \& Swift, S. 2002, ApJ, 576, 429

Silva, A. M., Azcarate, I. N., Poppel, W. G. L., \& Likkel, L. 1993, A\&A, 275, 510

Sopka, R. J., Olofsson, H., Johansson, L. E. B., Nguyen, Q.-R., \& Zuckerman, B. 1989, A\&A, 210, 78 
Su, K. Y. L., Volk, K., Kwok, S., \& Hrivnak, B. J. 1998, ApJ, 508, 744 Su, K. Y. L., Kwok, S., \& Hrivnak, B. J. 2000, Am. Astron. Soc. Meet., 197,0

Su, K. Y. L., Hrivnak, B. J., \& Kwok, S. 2001, AJ, 122, 1525

Su, K. Y. L., Hrivnak, B. J., Kwok, S., \& Sahai, R. 2003, AJ, 126, 848 te Lintel Hekkert, P., Caswell, J. L., Habing, H. J., et al. 1991, A\&AS, 90, 327

van de Steene, G. C., \& van Hoof, P. A. M. 2003, A\&A, 406, 773 van de Steene, G. C., Wood, P. R., \& van Hoof, P. A. M. 2000, in Asymmetrical Planetary Nebulae II: From Origins to Microstructures, ASP Conf. Ser., 199, 191

van der Veen, W. E. C. J., Habing, H. J., \& Geballe, T. R. 1989, A\&A, 226, 108

van Zadelhoff, G.-J., Dullemond, C. P., van der Tak, F. F. S., et al. 2002, A\&A, 395, 373
Weintraub, D. A., Huard, T., Kastner, J. H., \& Gatley, I. 1998, ApJ, 509, 728

Woods, P. M., \& Nyman, L.-A. 2004, A\&A, in prep.

Woods, P. M., Millar, T. J., Zijlstra, A. A., \& Herbst, E. 2002, ApJ, 574, L167

Woods, P. M., Millar, T. J., Herbst, E., \& Zijlstra, A. A. 2003a, A\&A, 402, 189

Woods, P. M., Schöier, F. L., Nyman, L.-A., \& Olofsson, H. 2003b, A\&A, 402, 617

Zijlstra, A. A., Chapman, J. M., te Lintel Hekkert, P., et al. 2001, MNRAS, 322, 280

Zuckerman, B., \& Lo, K. Y. 1987, A\&A, 173, 263 
P. M. Woods et al.: Molecules in bipolar PPNe, Online Material p 1

\section{Online Material}


P. M. Woods et al.: Molecules in bipolar PPNe, Online Material p 2

Appendix A: Molecular line spectra

Spectra of IRAS 16594-4656 and IRAS 17150-3224 taken with the SEST are presented in Figs. A.1-A.3. 
P. M. Woods et al.: Molecules in bipolar PPNe, Online Material p 3

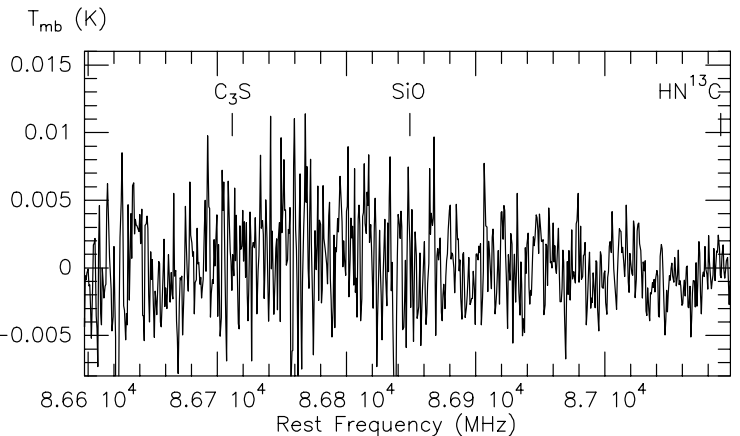

$T_{m b}(K)$

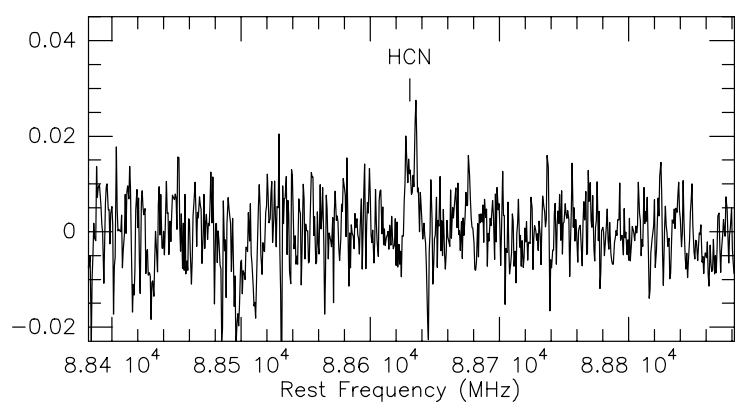

$T_{m b}(K)$

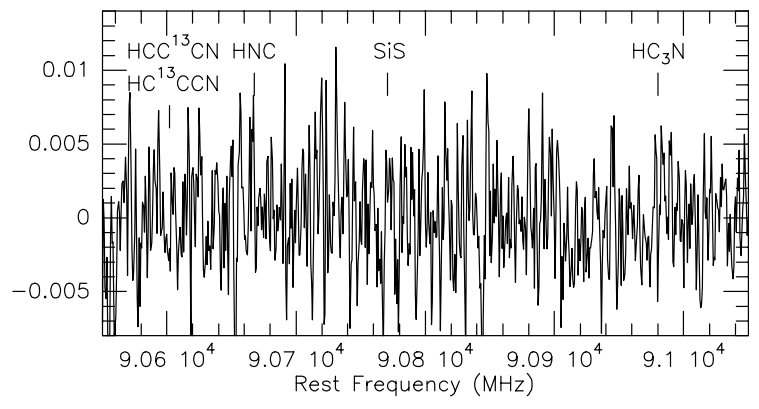

$T_{m b}(K)$

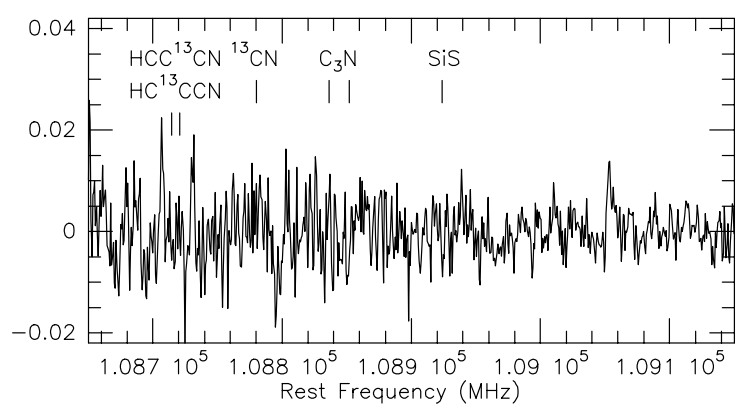

$\mathrm{T}_{\mathrm{mb}}(\mathrm{K})$

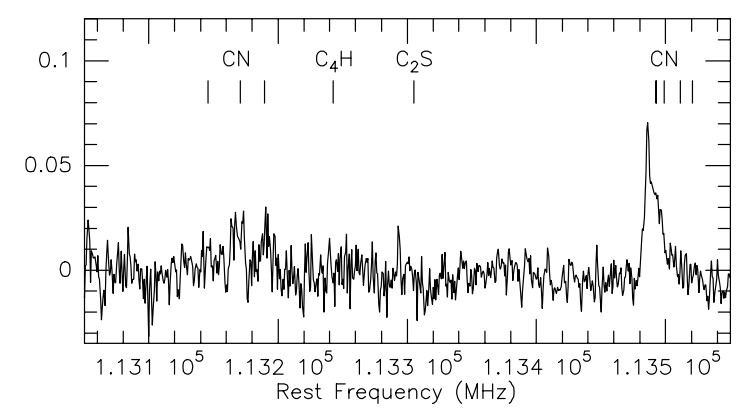

$T_{m b}(K)$
0.015

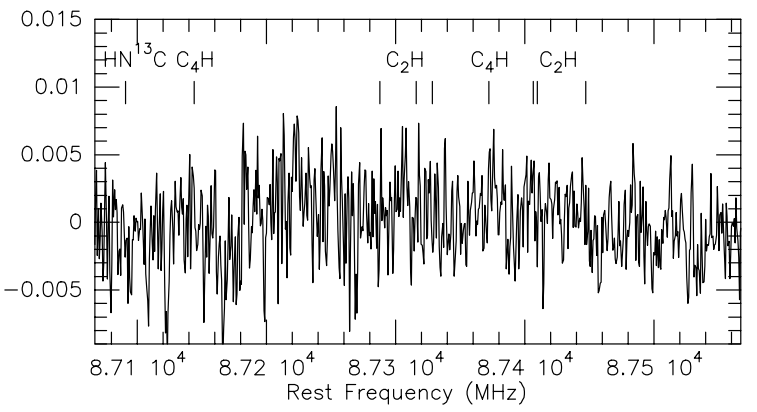

$T_{m b}(K)$

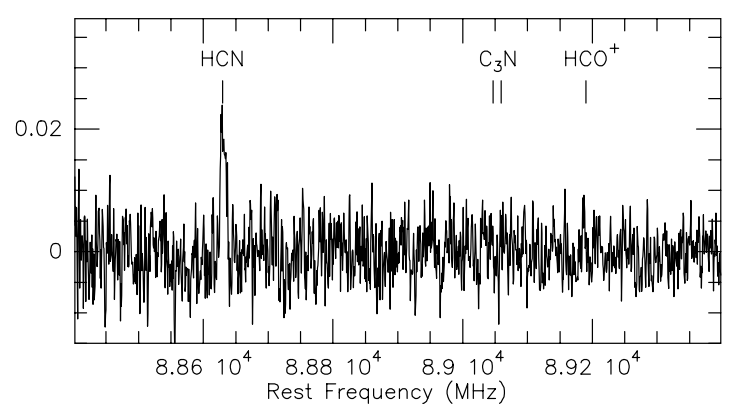

$\mathrm{T}_{\mathrm{mb}}(\mathrm{K})$

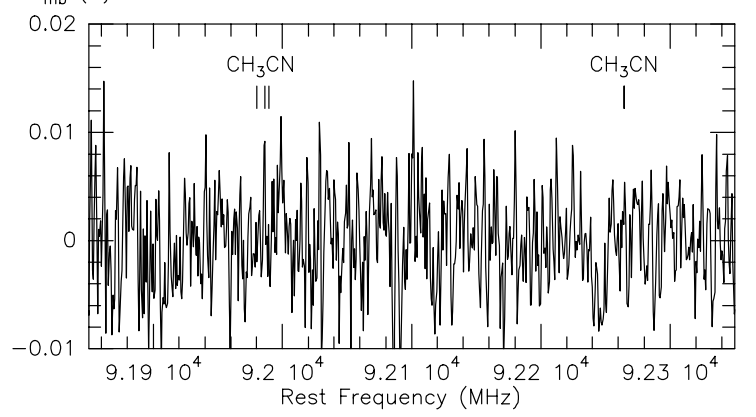

$T_{m b}(K)$

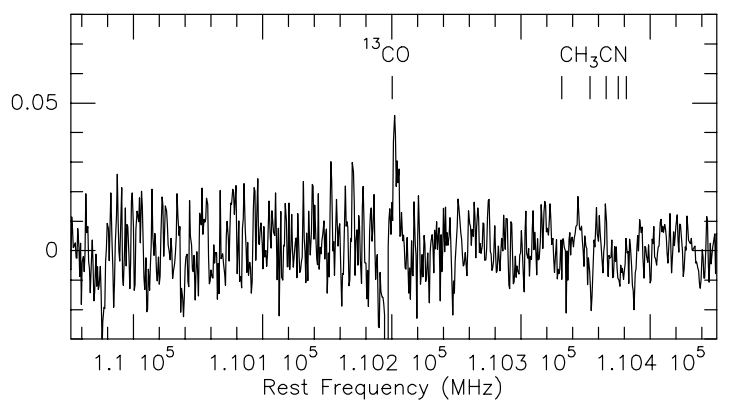

$T_{m b}(K)$

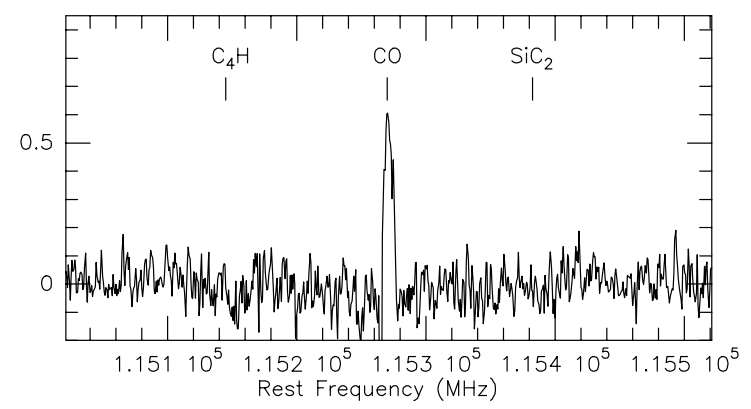

Fig. A.1. Molecular line observations of IRAS 16594-4656. 
P. M. Woods et al.: Molecules in bipolar PPNe, Online Material p 4
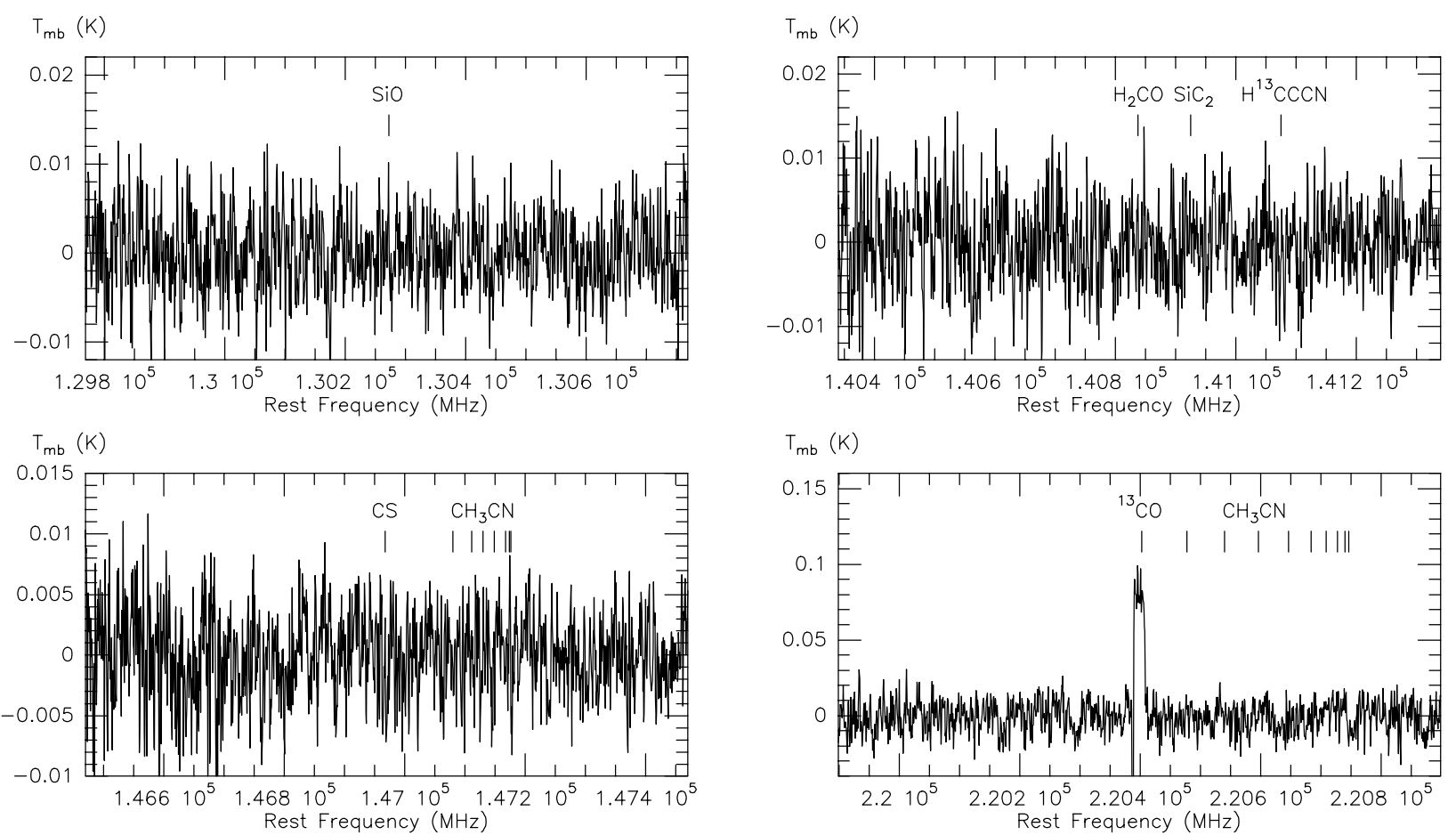

$$
T_{m b}(K)
$$

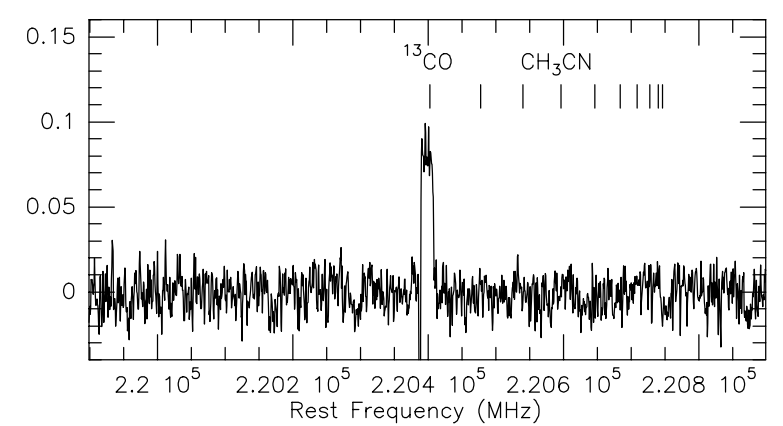

$T_{m b}(K)$

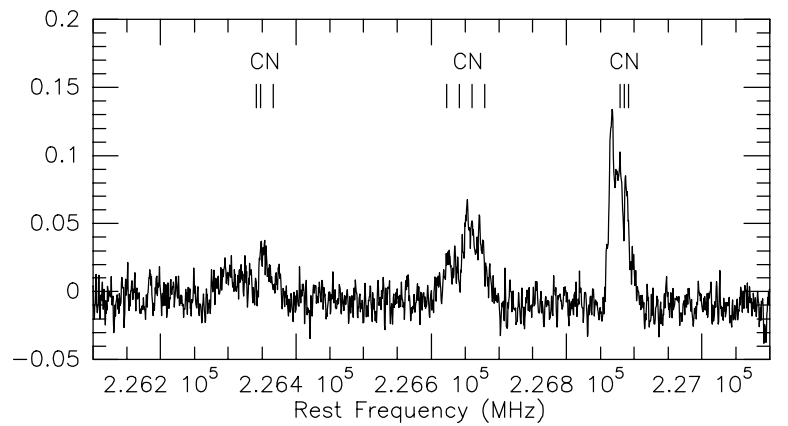

$T_{m b}(K)$

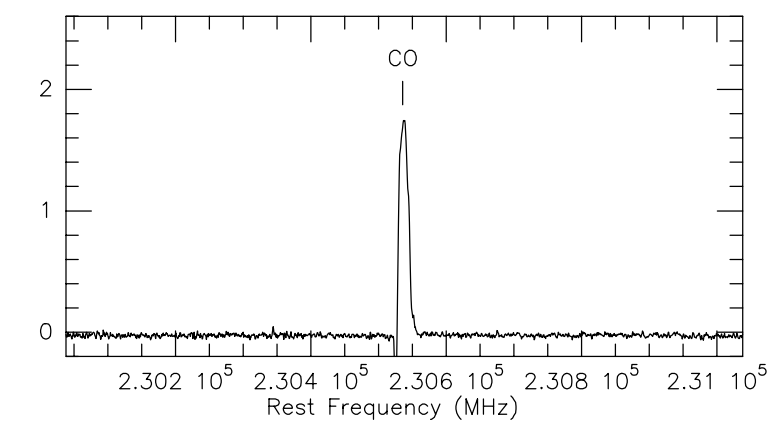

$\mathrm{T}_{\mathrm{mb}}(\mathrm{K})$

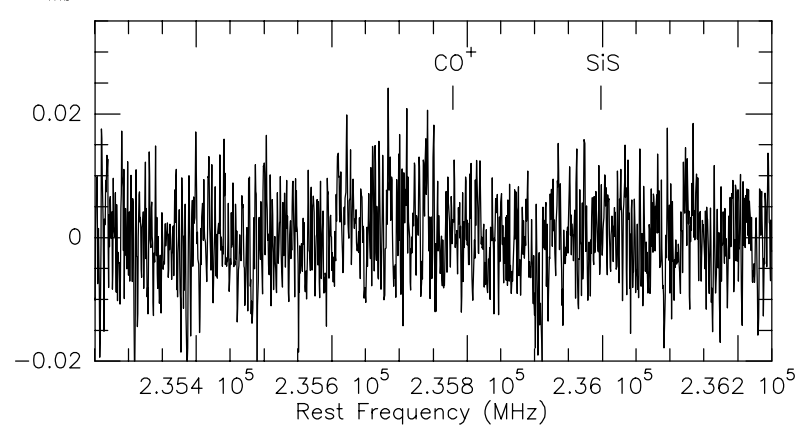

$$
T_{m b}(K)
$$

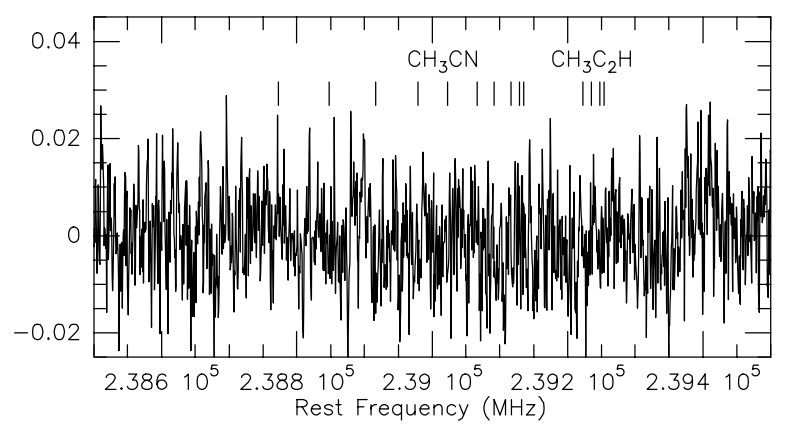

$T_{m b}(K)$
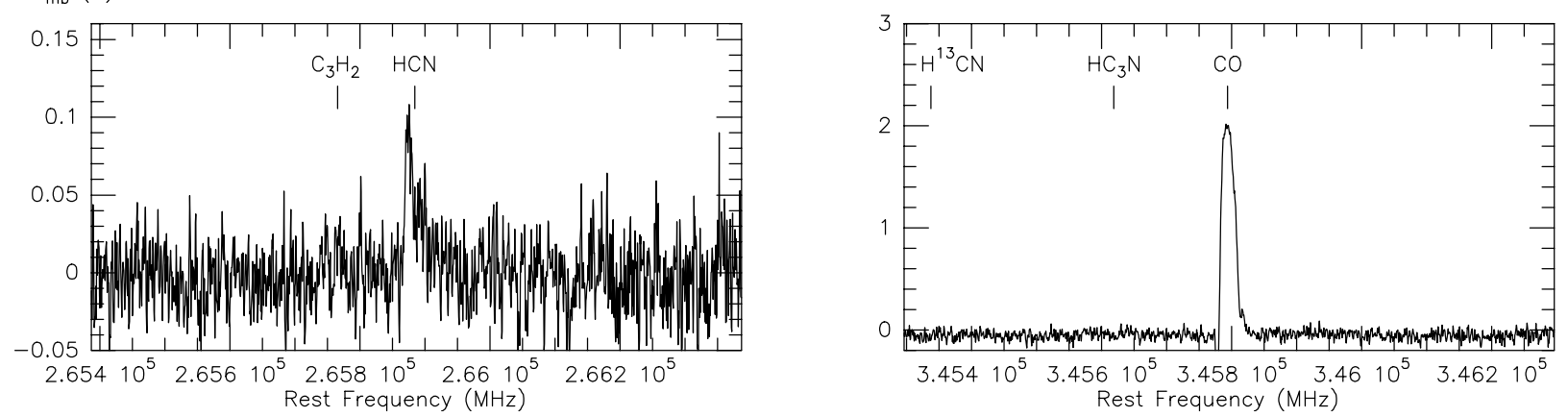

Fig. A.2. Molecular line observations of IRAS 16594-4656. 
P. M. Woods et al.: Molecules in bipolar PPNe, Online Material p 5
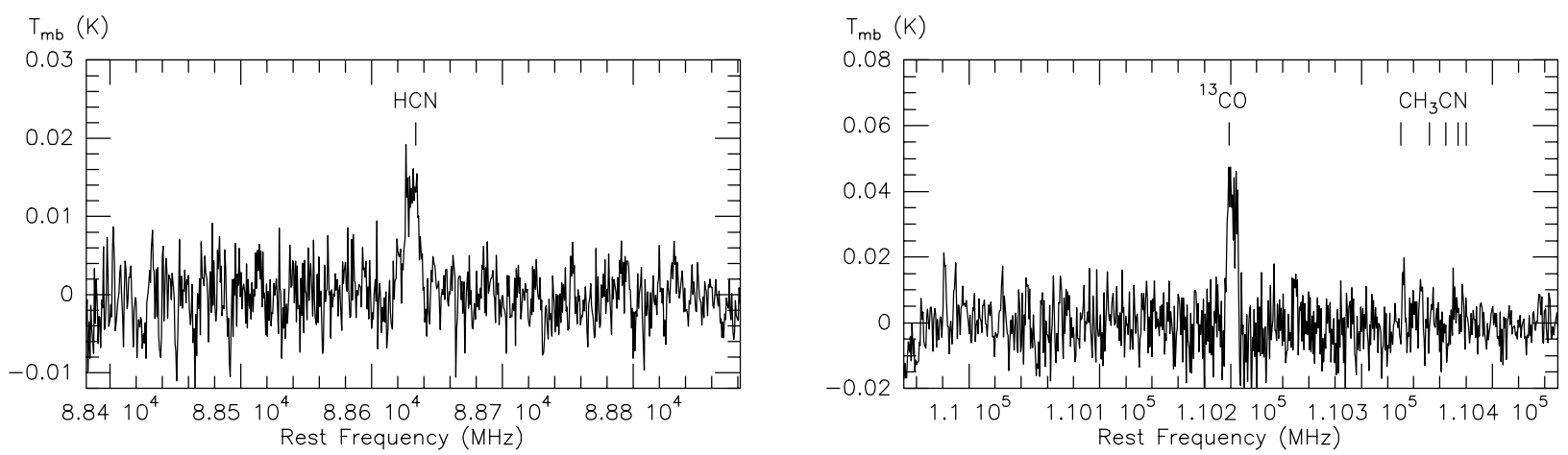

$T_{m b}(K)$

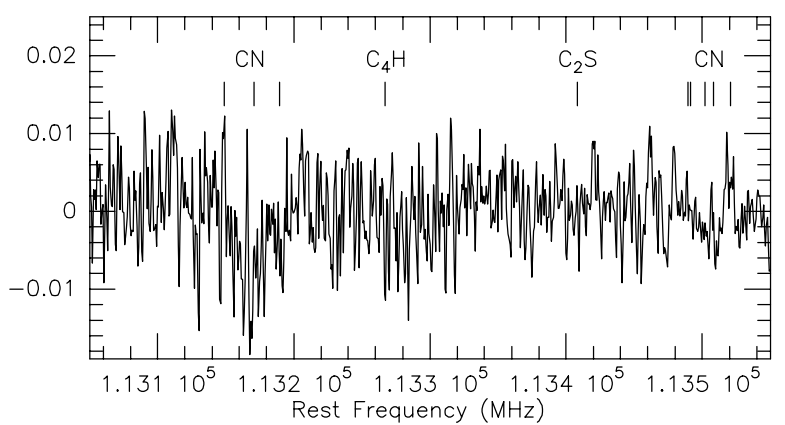

$T_{m b}(K)$
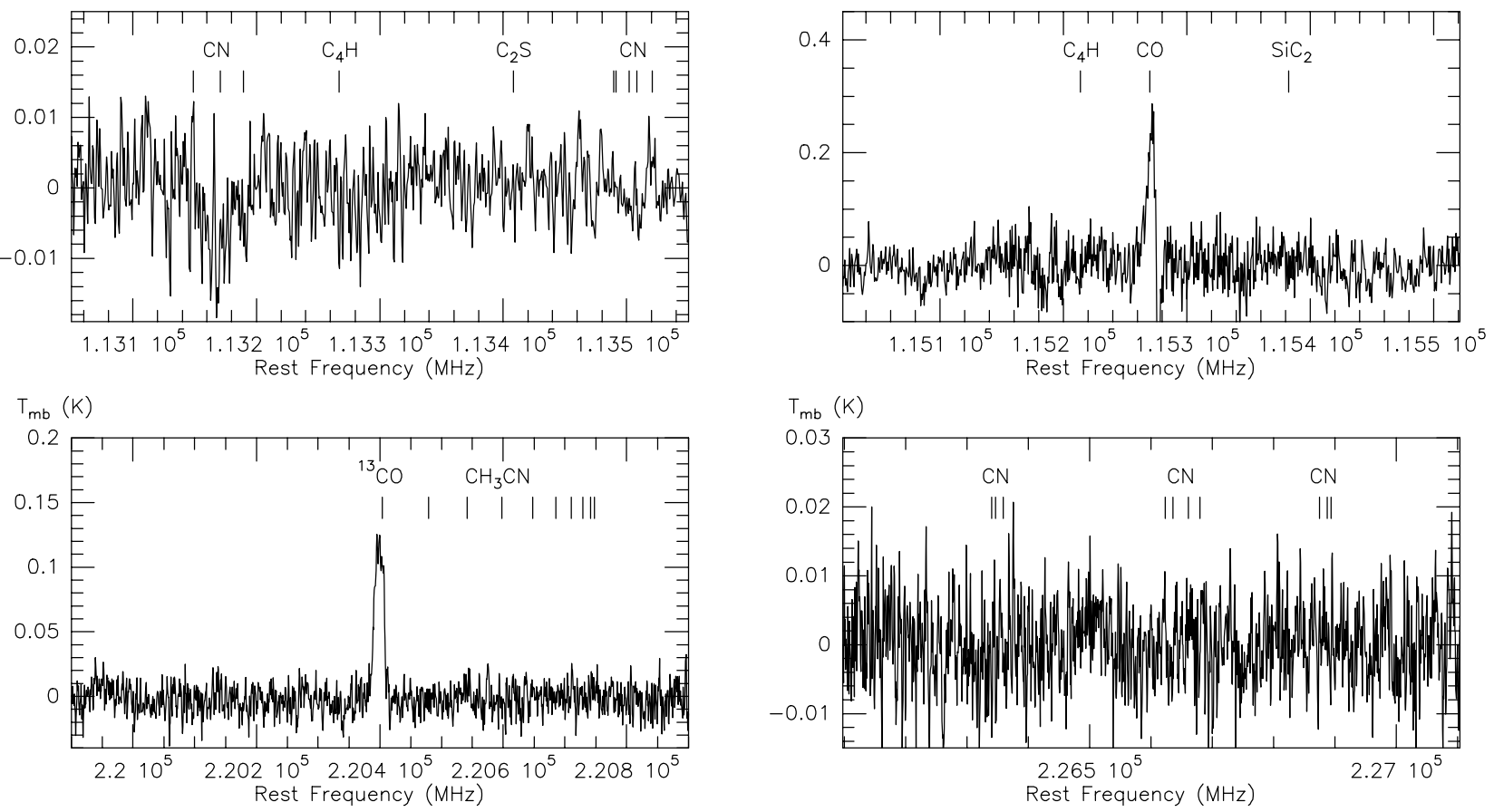

$T_{m b}(K)$

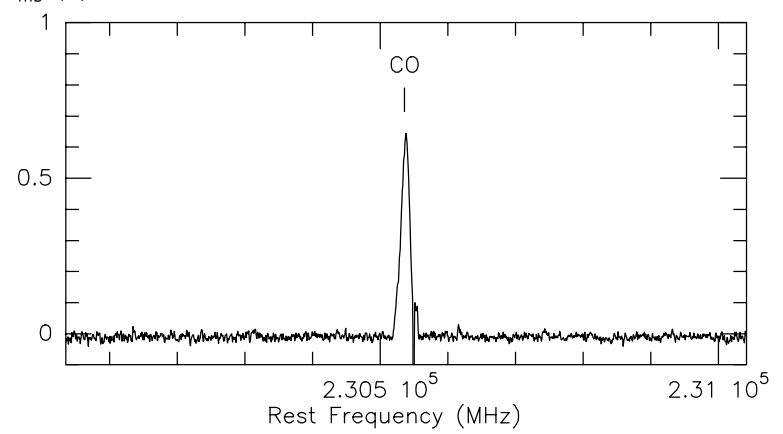

$\mathrm{T}_{\mathrm{mb}}(\mathrm{K})$

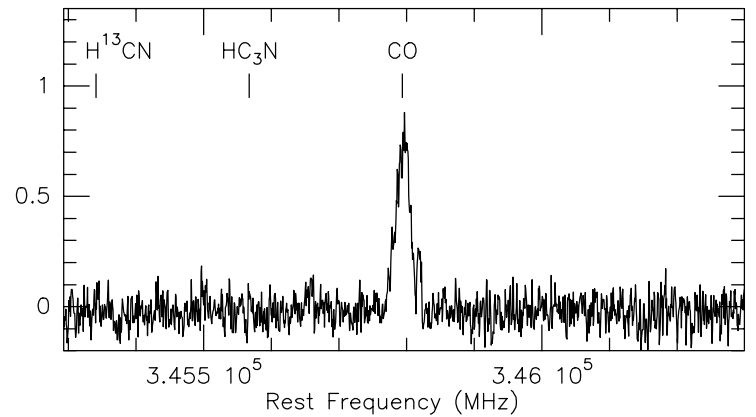

Fig. A.3. Molecular line observations of IRAS 17150-3224. 